SANDIA REPORT

SAND2000-8232

Unlimited Release

5 Printed April 2000

MS 9021

TECHNICAL COMMUNICATIONS

DEPARTMENT, 8528 FOR DOE/OSTI

$=\sqrt[3]{6}$

Capturing Recrystallization o
a Multi-Scale Materials Model
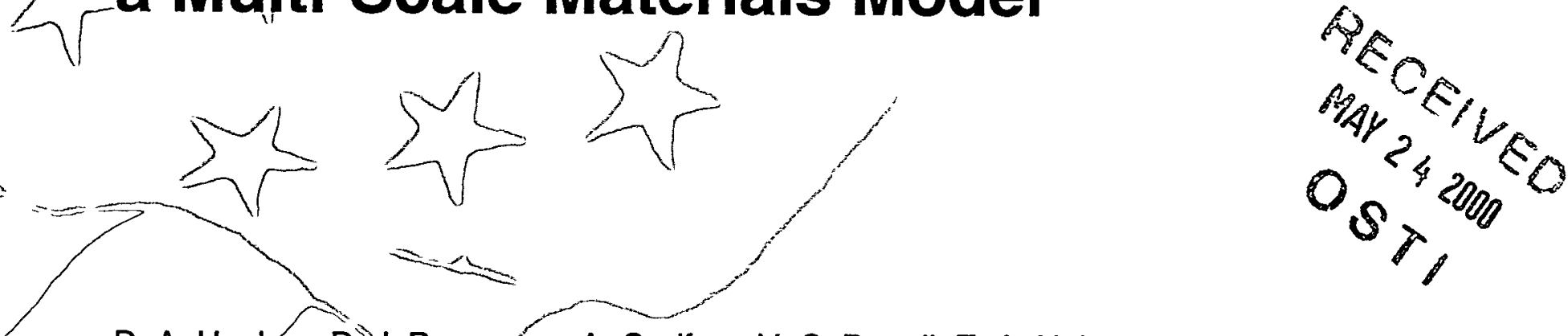

- D. C. Chrzan, M. T. Lusk

- Prepared by

Sandia National Laboratories

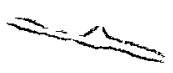

Metals with

Albuquerque, New Mexico 87185 and Livermore, California 94550

Sandia is a multiprogram laboratory operated by Sandia Corporation,

a Lockheed Martin.Company, for the United States Department of

Energy-under-Cóntract DE-AC04-94AL85000.

Approved for public release; further dissemination unlimited. 
Issued by Sandia National Laboratories, operated for the United States Department of Energy by Sandia Corporation.

NOTICE: This report was prepared as an account of work sponsored by an agency of the United States Government. Neither the United States Government, nor any agency thereof, nor any of their employees, nor any of their contractors, subcontractors, or their employees, make any warranty, express or implied, or assume any legal liability or responsibility for the accuracy, completeness, or usefulness of any information, apparatus, product, or process disclosed, or represent that its use would not infringe privately owned rights. Reference herein to any specific commercial product, process, or service by trade name, trademark, manufacturer, or otherwise, does not necessarily constitute or imply its endorsement, recommendation, or favoring by the United States Government, any agency thereof, or any of their contractors or subcontractors. The views and opinions expressed herein do not necessarily state or reflect those of the United States Government, any agency thereof, or any of their contractors.

Printed in the United States of America. This report has been reproduced directly from the best available copy.

Available to DOE and DOE contractors from

Office of Scientific and Technical Information

P.O. Box 62

Oak Ridge, TN 37831

Prices available from (703) 605-6000

Web site: http://www.ntis.gov/ordering.htm

Available to the public from

National Technical Information Service

U.S. Department of Commerce

5285 Port Royal Rd

Springfield, VA 22161

NTIS price codes

Printed copy: A05

Microfiche copy: A01

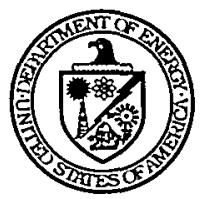




\section{DISCLAIMER}

Portions of this document may be illegible in electronic image products. Images are produced from the best available original document. 
SAND2000-8232

Unlimited Release

Printed April 2000

\title{
Capturing Recrystallization of Metals with a Multi-Scale Materials Model
}

\author{
D. A. Hughes, D. J. Bammann, A. Godfrey and V. C. Prantil \\ Science-Based Materials Modeling Department \\ Sandia National Laboratories \\ Livermore, CA 94551 \\ E. A. Holm \\ Materials and Process Modeling \\ Sandia National Laboratories \\ Albuquerque, NM 87185 \\ M. A. Miodownik \\ University College, Dublin, Ireland \\ D. C. Chrzan \\ University of California, Berkeley, CA 94720 \\ M. T. Lusk \\ Division of Engineering, Colorado School of Mines, Golden, CO 80401
}

\begin{abstract}
The final report for a Laboratory Directed Research and Development project entitled, Capturing Recrystallization of Metals in a Multiscale Materials Model is presented. In this project, deformation and recrystallization processes have been followed experimentally and theoretically in order to incorporate essential mechanisms from the defect (dislocation) and grain size length scales. A nonlinear rotational gradient theory has been developed which enables the incorporation of microstructural parameters. The evolution of these parameters during deformation and recrystallization has been characterized qualitatively and quantitatively, applying various electron optic techniques ranging over several length scales. The theoretical and experimental framework developed is general. It has been exemplified by an application to recrystallization in single crystals and bicrystals of aluminum. The recrystallization process has been modeled using a 3-D model for the changes in key structural parameters during recrystallization.
\end{abstract}


This page intentionally left blank. 


\section{Contents}

1. Introduction 7

2. Background and Motivation $\quad 7$

3. Scope 9

4. Defect Level 9

4.1 Experiments 10

4.2 Deformed State of Single Crystals and Bicrystals 13

4.3 Deformed Plus Annealed State of Single Crystals and $\begin{array}{ll}\text { Bicrystals } & 16\end{array}$

4.4 Summary of Microstructural Observations 17

$\begin{array}{ll}\text { 5. Grain Level } & 18\end{array}$

5.1 Polycrystal Plasticity (based on a contribution by V. Prantil)

5.2 Scaling Hypothesis

5.3 Modeling of Nucleation of Recrystallization (based on a contribution by M. Miodownik and E. Holm)

6. Continuum Level

6.1 Spatial and Rotational Gradient Continuum Level Material Constitutive Model (based on a contribution by D. Bammann)

6.2 Crystal Plasticity Model (based on a contribution by D. Bammann)

6.3 Phase Field Model of Recrystallization (based on a contribution by M. Lusk)

7. Achievements

8. Future work 
This page intentionally left blank. 


\section{Capturing Recrystallization of Metals with a Multi-Scale Materials Model}

\section{Introduction}

Material forming and shaping processes provide us with products ranging from commercial auto body panels and medical implants to DOE gas transfer systems (GTS). The success of this processing depends on the defect driven behavior of materials under mechanical and thermal loads, since this behavior controls the final structure and properties. Therefore a predictive continuum model for thermo-mechanical processing of metals needs to encompass essential mechanisms from the defect (dislocation) and grain size length scales.

In the following we describe the results of an LDRD project to develop a nonlinear-nonequilibrium-thermodynamic spatial gradient theory which provides the mathematical framework to incorporate key microstructural parameters. The parameters are obtained by a quantitative characterization of microstructure and local crystal orientation. Although the theoretical and experimental framework that is developed herein is quite general, we have chosen to apply this formulation to the analysis of recrystallization processes in metals.

\section{Background and Motivation}

Metals undergo recrystallization several times from the time they are cast until a metal part is fabricated. Recrystallization may occur during manufacturing, high rate deformation and/or in high temperature environments. Recrystallization is the process, occurring at elevated 
temperature, whereby new, nearly dislocation free grains nucleate and grow at the expense of old grains containing a deformation induced dislocation microstructure. Note that dislocations, i.e., the inherent defect structure of metals, provide the malleability necessary to form metals into complex shapes. The dislocation microstructure pattern that develops during processing directs the subsequent recrystallization. This pattern encompasses local lattice rotations that have a hierarchical scale of lengths. Thus following recrystallization, the new grains may have a different size, shape or preferred crystallographic orientation compared to the old grains. These new structures impart dramatic changes in yield stress, mechanical anisotropy and consequently material response during and after processing. See Figure 2-1 for a schematic illustration of this process.

The critical goal during processing and fabrication of metal parts is to control recrystallization and thereby tailor grain size, crystallographic texture, yield stress and mechanical property anisotropy. Since limited predictive capabilities exist, this goal is reached by trial and error processing which for a single product require reprocessing of large tonnages of metals. The ultimate goal of this research is to create a predictive continuum model that correctly incorporates evolving microstructures and texture so that we can tailor both processing and resulting structure. This tailoring is desired to improve process efficiency, reliability and performance.

The continuum model is nonlocal so that the identification and derivation of representative length scale state variables are essential to its predictive capability. The length scales must not only be consistent with continuum theory but also must be motivated by and embody the effects of the experimentally observed microstructures. Additional information on the appropriate length scale variables at the grain level are required. For this purpose meso-scale analytical models, e.g., scaling theory and numerical models, including phase field, Monte Carlo Potts and polycrystal plasticity models are used.

As a specific application, Sandia is responsible for manufacturing and process modeling of forged weapon components, including nose cones, cases, and gas transfer systems. Captive manufacturers previously performed expensive trial and error processing to meet our stringent grain size and grain shape requirements. Due to the economics of small batch manufacturing, our new, commercial suppliers will have difficulty meeting our requirements with reasonable costs, unless we can provide them with a rapid design tool. For specific examples of problems controlling the recrystallization process during commercial forging of GTS components see Robinson et al., 1999. 


\section{Scope}

Recrystallization involves microstructural evolution and physical processes over a wide range of length and time scales. Physical models and experimental evidence is now emerging to bridge these scales. Four major aspects of this problem were selected as a focus for this research:

1) Capturing the experimentally observed dislocation microstructure pattern quantitatively across several size scales for input into material models.

2) Incorporating this observed microstructure into a grain level model.

3) Developing a grain nucleation model based on the dislocation microstructure with the added considerations of stored energy, boundary energy, boundary mobility and crystallographic texture.

4) Deriving a spatial gradient based continuum level material constitutive model that would provide multiple length scales and rotational gradients.

Figure 3-1 shows a flow chart of the ingredients necessary to create a recrystallization model as well as the end use of these results. Note that these results can feed either directly into process wisdom for forging GTS components or indirectly through future implementation into a FEM code like JAS 3-D.

\section{Defect Level}

Several experiments in this LDRD program focussed on creating and measuring the development of dislocation microstructure patterns during deformation, since these patterns will direct any subsequent recrystallization. Microstructural observations were performed over a wide range of scales from nanometers to several millimeters following both deformation and deformation-plus-annealing. This range of observations had the aim of revealing the hetereogeneity and hierarchies in the deformation patterns across a sample as well as understanding the role of the basic microstructural unit.

Since for many metals and alloys an arrangement of dislocations into nearly two dimensional boundaries with dislocation poor regions in between is the characteristic feature of the microstructure, we take the dislocation boundary as our basic microstructural unit. Thus we are particularly interested in defining microstructural parameters based on the spacing, spatial distributions, and morphology of these deformation-induced boundaries. 
Additionally these boundaries separate regions of the crystal that are rotated away from each other. Since the regions adjacent to the boundary have somewhat different crystal orientations, another parameter associated with the boundary is its misorientation angle. The last parameters that are needed are the local crystal orientations that develop during deformation and the spread of these orientations across a sample. These last parameters are related to crystal plasticity.

A new experimental capability was implemented on our field emission transmission electron microscope (TEM) to provide semiautomatic in situ measurements of these microstructural parameters. This microstructural information was then distilled using analytical tools for incorporation into the grain and continuum level models to be described in chapters 5 and 6 respectively. Samples with selected microstructures were subsequently annealed to produce partly and fully recrystallized microstructures. Thereby, relationships between the deformed and recrystallized microstructures could be examined and strategies developed for the mesoscale nucleation and recrystallization models.

\subsection{Experiments}

\subsubsection{Materials, Mechanical Testing and Microscopy}

A well defined deformation pattern was obtained by selecting samples in the form of single crystals and bicrystals. The single crystal had an orientation aligned for glide on one primary slip system. One crystal in the bicrystal (Figure 4-1) had this same orientation as the single crystal.. The other was in a different orientation but also aligned for single slip. This choice resulted in well defined microstructures which could create evidence for different nucleation events following recrystallization heat treatment after deformation.

High purity (>99.99\%) aluminum bicrystals were grown for us by Metals, Oxides and Crystals Ltd., Oxford, U.K., to have a $\Sigma 29,43.6^{\circ}[100]$ bicrystal boundary (Figure 4-1). The as-grown crystals were not perfectly oriented; growth errors resulted in a boundary angle of $41.6^{\circ}$ [100 5 8] instead of $43.6^{\circ}$ [100]. More information regarding the designation of this special boundary and bicrystal is given in Appendix $\mathrm{A}$.

Compression samples were electro-discharged machined (EDM) from the single crystals and the bicrystals to have the configuration shown in Figure 4-1. Note that the [421] crystal direction of the single crystals and one of the crystals in the bicrystal is parallel to the cylinder/compression axis. The other crystal in the bicrystal had a [104 $\overline{3} \overline{1} 18]$ orientation. Orienting the bicrystal 
in this manner resulted in the bicrystal boundary being inclined $12.6^{\circ}$ from the compression plane as shown in Figure 4-1.

Both the single and bicrystals were compressed at $\dot{\varepsilon}=.001^{\mathrm{s}-1}$ to different strain levels including $\varepsilon_{\mathrm{vM}}=0.15,0.2,0.3,0.45$ and 0.6 . Friction was minimized during compression using Teflon sheets and $\mathrm{MoS}_{2}$ lubricant. No barreling of the single crystal or polycrystal samples was observed following von Mises strains of 0.6 .

After compression samples were sectioned by EDM parallel to the cylinder axis. Some sections were left as-deformed while others were also annealed to either partly or fully recrystallize the crystals. The microstructures in the asdeformed and as-deformed-plus-annealed sections were examined by optical metallography, scanning electron microscopy including automatic electron back scattered pattern analysis of crystal orientations, and transmission electron microscopy with Kikuchi pattern analysis of crystallite orientations. All deformed samples were kept in a freezer when not being examined to avoid recovery of the highly deformed high purity aluminum.

\subsubsection{Development of New Experimental Capabilities for the FETEM}

As part of this program, a new experimental capability had to be developed and implemented for the newly arrived field emission TEM. The need for statistically large numbers of microstructure measurements in our experimental program demanded an semi-automatic technique. Computer control of the new TEM and specimen goniometer enabled us to implement a semi-automatic technique for in situ measurement of crystal orientation, calculation of boundary misorientation angles, pole figures and crystal planes based on Kikuchi pattern analysis. While the principles of this technique originate with our close collaborators Liu, 1994, we have implemented a faster, broader ranging and more automatic capability that is unique to our facilities at SNL, CA. This capability allows us to obtain statistically significant distribution of deformation boundary misorientation angles for a given condition.

\subsubsection{Development of New Stereological Techniques for Measuring Dislocation Boundary Spacing Distributions.}

Determination of the dislocation boundary spacing distribution is not as straightforward as for the boundary misorientation angle distributions. The boundary misorientation angles are calculated from orientation measurements, which are a single point statistic and therefore can be measured directly in any section. The requirement of investigating deformation microstructures using thin foils in the TEM is such that we are 
limited to the observation of a projection onto a plane of a three-dimensional structure. It is necessary therefore to consider the stereology in order to obtain the dislocation boundary spacing distributions.

In particular, extended nearly planar dislocation boundaries, that are an important feature of the deformed microstructures, form an example of what have been termed oriented structures, the stereological analysis of which has been discussed both by Underwood, 1970 and DeHoff and Rhines, 1969. The majority of existing analyses of such structures are concerned only with determining average properties, and although Cahn and Fullman, 1956 have developed an elegant model for the determination of true spacing distributions, this requires either multiple random sectioning or a single section through many colonies, neither of which is appropriate for analyzing the extended dislocation boundaries. In ideally oriented structures (those where the planar features are all parallel) the linear relationship between the spacing in a section and the true spacing allows the determination of true spacings, if the inclination angle from the perpendicular direction can be measured. Note that this problem is simplified if one normalizes a distribution by it average.

However, for real dislocation structures the relationship is further complicated by the fact that the walls are only nearly parallel. Additionally one is limited in general (for polycrystals) to examining a single section through any given structure, where the thin area only allows a limited (statistically low) number of measurements. The problem is therefore both one of stereology and of statistics.

In order to examine the combined effect of these factors a geometric computer model has been developed and utilized, whereby sets of nearly parallel virtual planes can be constructed, sectioned and analyzed, see Godfrey and Hughes, 1998 and 2000. This method allows the comparison of distributions garnered in different sections through the same structure. Results from this model suggest that for sets of planes that are nearly parallel to one another, the true distribution shape can be sufficiently determined by making measurements of apparent spacings in any section that intersects the planes with angle $>30^{\circ}$, providing a wide margin of error. This method is more fully described in references Godfrey and Hughes, 1998 and 2000.

Note that in the experiments herein, the spacings measured are very close to the true perpendicular spacing, since either the TEM foils were tilted to orient the boundaries perpendicular to the beam or sections were chosen in which the boundaries are most nearly perpendicular to the foil surface. 


\subsection{Deformed State of Single Crystals and Bicrystals}

Observations were made with different methods to uncover the hierarchies of the microstructural patterns. Of special interest is the extent to which the bicrystal boundary changes the dislocation microstructure and grain subdivision as well as the distance over which the boundary influences the structure. A number of features were measured to enable comparison of experiment with the different level models

\subsubsection{Sample Scale Observations: External Shape Change}

The macroscopic shape change of the compression samples during deformation is used to gage the average distribution of the slip pattern, providing one measure to assess crystal plasticity and grain level models. For the [421] single crystal the original shape of the right circular cylinder was altered during deformation to that of a cylinder with an oval cross section and a slight shear of the sides (Figure 4-2, $\varepsilon=0.6$ ). The long axis of this oval is parallel to the projection of the primary slip direction in the compression plane. All lateral extension of the sample was along the long axis of this oval. No extension occurred along the short axis. This macroscopic shape change is consistent with a prediction of single glide on average.

Each crystal of the bicrystal was also oriented initially for single glide, but with their primary slip directions $31^{\circ}$ apart on respective slip planes that are inclined $37^{\circ}$ from each other (Figure 4-1). The projections of these slip directions in the compression plane are $50^{\circ}$ apart. This incompatibility of slip within the bicrystal affected the shape change of the bicrystal during deformation. The distorted shape of the bicrystal deformed, $\varepsilon=0.3$, is shown in the macrophoto, Figure 4-3. The deformed shape is roughly like stacking two oval cylinders on top of each other with their long axes twisted away from each other by $42^{\circ}$. More specifically, away from the bicrystal boundary, the cross-section of the [421] crystal half is nearly the same oval shape as in the single crystal with the long axis along the projection of the primary slip direction. This shape is mimicked in the second [104 $\overline{3} \overline{1}$ 18] crystal albeit with the long axis of its oval oriented near the projection of its respective primary slip direction. As the oval cross sections are viewed in either crystal, closer and closer to the boundary, their aspect ratios gradually decrease, allowing the ovals to blend together near the boundary in a compromise shape and direction (Figure 4-3). This shape change suggests that slip differences imposed by the constraint of the boundary are spread over broad distances, on the order of millimeters, within the sample.

At ever increasing strains, e.g., $\varepsilon=0.3$ to 0.6 , the shape change of the compression sample results in the unloading of the over hanging portions of 
the mismatched oval cross-sections. This unloading in turn affects the subsequent development of the shape change and deformation state in the compression samples.

\subsubsection{Sample to Defect Scale Observations: Microstructure}

The shape change of the bicrystal suggests that the deformation was not uniform within the bicrystal, whereas that for the single crystal suggested more homogeneous deformation. The degree of heterogeneity is revealed by looking at the microstructures.

\subsubsection{Hierarchical microstructure.}

Optical metallography, SEM back scattered imaging and TEM revealed a macroscopic subdivision of the single crystals and bicrystals into regions with different crystal orientations, e.g., Figure 4-4. Long nearly sample scale bands are observed. The bands range from about $5 \mu \mathrm{m}$ to $100 \mu \mathrm{m}$ wide by $100 \mu \mathrm{m}$ to $4 \mathrm{~mm}$ long. Note that the size of these bands is often larger than a typical grain in a polycrystal. The coarsest of the bands observed in the TEM could also be seen by SEM and optical techniques.

When these bands and their surroundings are viewed in either the SEM by channeling contrast or in the TEM, it is observed that these bands have no sharp single boundary (Figure 4-5). Rather they comprise regions with a distinct dislocation microstructure. The dislocation microstructure within these bands is composed of two intersecting families of geometrically necessary dislocation boundaries (GNBs). In contrast, in the matrix surrounding these bands, the dislocation structure is composed of a single family of GNBs nearly parallel to the primary slip plane. An example of these two regions is shown in the TEM micrograph and sketch Figure 4-6 for the compressed [421] single crystal. This structure is comparable to those observed in the [421] side of the bicrystal. The structure within the matrix [104 $\overline{3} \overline{1} 18]$ side is shown in Figure 4-7. In the [104 $\overline{3} \overline{1}$ 18] side of the bicrystal the deformation microstructure in the matrix region are also composed of a single family of GNBs nearly parallel to the primary slip plane.

Curiously, From Figure 4-8 it can be shown that the dislocation structures on either side of the bicrystal boundary do not appear to be influenced much by the boundary. Only a subtle effect is observed very near the bicrystal boundary in which a few GNBs curve slightly as they approach the boundary (see arrow).

These micrographs illustrate that nearly planar and extended nature of the GNBs. These extended boundaries enclosed groups of cells in cell blocks. The 
cells are more or less equiaxed regions of crystal containing few dislocations that are surrounded by incidental dislocation boundaries (IDBs)(a.k.a., cell walls).

For these crystals and bicrystals three levels of subdivision were observed including at the highest level long macroscale bands in a matrix structure, GNBs forming cellblocks at the next level and cells at the smallest. Three levels of subdivision are frequently found in single crystals (Becker et al., 1991, Godfrey et al., 1998, Liu and Hansen, 1998).

\subsubsection{Origin of the macroscale banding.}

It is suggested that the bands form in the single crystal primarily to accommodate the deformation constraint imposed by the compression platens. While friction was very low, as determined by the sample shape change, these conditions are not frictionless. Thus it is possible that the very low level friction may make a small contribution to strong constraint provided by the platens. (Wonsiewicz and Chin, 1970, Liu and Hansen, 1998).

Since macroscopic bands form in both the single and bicrystals, the formation of the macroscopic bands is not simply the result of the bicrystal boundary. Instead, the macroscopic breakup of the crystals is primarily the way in which the crystals accommodate the deformation constraint from the external applied boundary conditions rather than the internal bicrystal boundary. The differing accommodation required by the bicrystal compared to the single crystal is met by a differing distribution of the macroscopic bands across the crystals. These differences include the location, spacing, length, width and macroscopic orientation of the bands with respect to the sample axes, Figure 4-4. Note there are several families of nearly parallel bands with different macroscopic orientations. Some bands are very coarse and long; others are very fine. While some of these bands intersect the bicrystal boundary they are not observed to penetrate across the boundary in the sections observed thus far.

\subsubsection{Quantitative measurements of the microstructure}

\subsubsection{Local crystal orientations}

The macroscopic regions represent regions of differing secondary slip as evidenced by the change in orientation within the cell blocks and by the larger misorientations across dislocation boundaries within the bands compared to those in the matrix regions. Examples of the color maps of the different orientations within cross-sections of the deformed bicrystals, as measured by SEM EBSP, are shown in Figure 4-9. The bands that were 
observed by optical contrast in Figure 4-4, appear in the orientation map as regions whose average orientations differ from the matrix by 10 to $30^{\circ}$.

The color maps Figure 4-9 show that there is not a monotonic gradient of slip in a direction away from the bicrystal boundary. Slip pattern changes that produce these macroscopic bands create the means for the crystal to spread the necessary accommodation required by the compression platens and the bicrystal boundary over large areas across the sample. These orientation changes due to slip pattern differences appear to vary from band to band to accommodate the deformation constraints.

\subsubsection{Misorientations across dislocation boundaries.}

GNBs: Local orientations measured in the TEM for adjacent regions separated by an individual GNB showed that much larger boundary misorientation angles, of 3 to $20^{\circ}$, developed for GNBs within the macroscopic bands compared to 1 or $2^{\circ}$ within the matrix following compression, $\varepsilon=0.3$. In general the average misorientation angle increases with increasing strain for each region, Godfrey and Hughes, 2000.

The IDBs (cell walls) develop smaller misorientation angles on average compared to the GNBs, Hughes et al, 1997, Godfrey and Hughes, 2000. For example at a strain of 0.3 in the [421] single crystal the average for IDBs is $0.5^{\circ}$ compared to $1^{\circ}$ for the GNBs.

\subsubsection{Spacing of dislocation boundaries}

The average true spacing of GNBs decreased with increasing strain, Godfrey and Hughes, 2000. The bicrystal and the single crystal had similar average boundary spacings of $2.7 \mu \mathrm{m}$ at a strain of 0.3 . The spread of sizes ranges from a small fraction of the average to about three times the average.

Distributions showing the spread of GNB spacings within a region are shown in Figure 4-10.

\subsection{Deformed Plus Annealed State of Single crystals and Bicrystals}

The deformed structure of both the high purity aluminum single crystals and bicrystals was shown to consists of macroscale bands in layers throughout a sample. This layered structure strongly directed the development of the annealed and recrystallization microstructures during heat treatment. This development can be followed by considering the [421] single crystal compressed to $\varepsilon_{\mathrm{vM}}=0.45$ and subsequently annealed at $150^{\circ} \mathrm{C}$ for different times and then quenched to room temperature. 
The microstructure inside these macroscale bands has a high stored energy and contains deformation induced high angle boundaries, whereas the matrix on either side of the bands has a much lower stored energy and contains only low angle boundaries. The macroscale bands thus have a structure that is much more conducive to nucleation and recrystallization. Consequently, a two step recrystallization process is observed in these layered structures as follows (Figure 4-11).

1) After one to ten minutes at $150^{\circ} \mathrm{C}$, nucleation of new grains only occurs within the macroscale bands. This nucleation occurs both within and at the edge of the bands. Nucleation may occur by several processes either in isolation or combination. These processes are subgrain growth, subgrain coalescence and strain induced boundary migration (SIBM), see the schematic Figure 4-11 $\mathrm{a}$ and $\mathrm{b}$ and optical micrograph Figure 4-12. The nuclei grow along the length of the macroscale bands thereby creating a bamboo structure.

2) These nuclei /grains thereby reduce the stored energy within the macroscale bands creating the potential for the high angle boundaries to migrate from the macroscale bands into the matrix regions by SIBM, Figure 4-11c.

Annealing at $325^{\circ} \mathrm{C}$ results in full recrystallization of both the deformed, $\varepsilon_{\mathrm{vM}}=0.45$, single crystal and bicrystal into coarse grained polycrystals. The growth of new grains removed the original bicrystal boundary. Boundary etching in the salt bath during annealing revealed that the growing grains had swept across the original bicrystal boundary. Although no trace of the original bicrystal boundary was observed, some new grains had grain boundaries whose morphology, position and inclination matched that of the macroscale bands.

The observations indicated that the presence of the macroscale bands with high misorientations were stronger nucleation sites than the original bicrystal boundary. This result is in accord with the observations in the cold deformed stage showing that gradient of stored energy in the region around the boundary in the bicrystal was not very large.

\subsection{Summary of Microstructural Observations}

Deformation in both the single crystal and bicrystal creates three levels of subdivision as an integral part of the slip pattern and microstructure. The macroscale subdivision into sample scale bands is the means by which the 
single crystal accommodates the constraint of the compression platens. Analogously in the bicrystal, the constraint of the bicrystal boundary is simply accommodated by adjusting the distribution of these macroscale bands. Consequently, instead of slip differences being concentrated at the bicrystal boundary, this macroscale subdivision results in a more even spread of slip differences extending away from the boundary for about half of the sample.

This macroscale banding directed the subsequent nucleation and recrystallization upon annealing.

Optical (shape change) and SEM (orientation determination) investigations have shown convincingly that distinct patterns of deformation occur on the macroscopic scale, providing data at a length scale suitable for examination using current crystal plasticity models.

\section{Grain Level}

\subsection{Polycrystal Plasticity}

The observed behavior on the sample to local scale provides the background with which to characterize and assess crystal plasticity models. Calculations have been performed on two finite element models of the bicrystal. Both a coarse (approximately 1000 elements) and moderately refined (approximately 16,000 elements) discretization corroborate the shape changes sufficiently removed from the bicrystal boundary.

Fully three-dimensional bicrystal finite element models were analyzed in quasi-static compression using an implicit, velocity-based finite element code, FEM-EVPS (Dawson and Marin, 1998; Marin and Dawson, 1998). As no general mesh generating capability was available for FEM-EVPS, a 3D 8node, hex element mesh generator was developed specific to cylindrical specimens with generally inclined bicrystal boundaries. High purity aluminum compression data was fit to $30 \%$ strain using a standard slip system power law constitutive relationship with a Voce hardening law (Figure 5-1). A initial investigation was performed beginning with a relatively coarse mesh discretization of 1024 three-dimensional, 8-node hex (brick) elements as shown in Figure 5-2. While the mesh is admittedly not sufficiently refined to capture the crystal misorientation patterns that are observed, it served as an initial test case to examine if the crystal plasticity discrete slip model appropriately resolved slip patterns in sufficient detail, on 
average, to capture the overall macroscopic shape change of the bulk specimen.

\subsubsection{External Shape Change}

Subsequent analysis indicated this appeared to be the case for even this simple mesh. In Figure 5-3, the experimental bicrystal shape is compared to the model at $15 \%$ compression. The model indicates the end face ovals in a direction parallel to the projection of the primary slip direction onto this end face. This observation is consistent with expectations if the crystal plasticity model captures distinct slip behavior on average over a collection of enough crystals with a single initial orientation, as is the case in each half of the bicrystal model. In the vicinity of the bicrystal interface, the major axis of ovalling is in a direction between the projected primary slip directions in each individual crystal on either side (Figure 5-4). This supports the fact that crystal plasticity models resolve the primary slip system activity reasonably well. The next section will address the more significant evidence of slip system activity, the crystal orieantion evolution.

In a limited effort to begin addressing mesh resolution, we generated a 16,384 element bicrystal (8192 hex elements per crystal) about a $\Sigma 29$ bicrystal boundary and compressed the model to $15 \%$ strain. The mesh is pictured in Figure 5-2. The shape change, as illustrated in Figure 5-5, is very similar to predictions with even the coarse mesh. While this is an appealing result, it is not entirely surprising given that it is generally accepted that average behavior is often predicted by crystal plasticity models when the number of crystal is large enough for the average measures to be statistically significant. Thus, based on the overall shape change, the average texture appears to be predicted well.

\subsubsection{Crystal orientation evolution}

With tens of thousands of individual crystal orientations updated at each time increment, the crystal plasticity finite element model simulations provide information on fields of relative lattice misorientation that may be used to compare with experimental measurements of local crystal orientation in the TEM and SEM (Figure 5-6). Even with this mesh resolution, however, the rotation direction of the crystals and the relative spread of the texture does not appear to be predicted by the standard models (Figure 5-6). Differences in the ability of the model to predict the crystal orientations, depend on which crystal side of the bicrystal is considered. The model predicts the rotation direction of the [421] crystal side, but fails to match the very wide spread in rotation, $40^{\circ}$, along this rotation path that is observed in experiment. In contrast, the rotation directions of the [104 $\overline{3} \overline{1} 18]$ crystal 
side in the experiment are completely different than those in the model. It appears that the bicrystal boundary creates a condition that equally activates the first two highly stressed slip systems. This second system is one that the model does not sufficiently activate.

While this appears to be true for the particular modeI analyses presented here, there may be over riding reasons for this, which could prevent one from making more broad generalizations based on this limited set of analyses.

First, we have not, apart from mesh discretization, altered or varied what may be significant features of the model to which our predicted misorientation fields may be sensitive. These may include, but are not limited to, perturbations in the initial texture, material rate sensitivity, single crystal hardening laws, and the far-field boundary conditions (both fixed and friction assumptions).

Additionally this model is designed for constrained polycrystals rather than the more limited constraint of a bicrystal. More importantly, however, it may be necessary to introduce more realistic slip system kinetics to predict slip system activity that results in the orientation evolution observed experimentally in the $\left[\begin{array}{llll}104 & \overline{3} & \overline{1} & 18\end{array}\right]$ crystal side (Figure 5-6a).

Second, the mesh discretization study, itself, is far from complete. Even the relatively refined mesh used here allows for only a marginal number of elements within a single crystal to resolve misorientation band spacing observed in the TEM. We had been somewhat restricted, however, by the computational intensity of the calculations at even this scale on a serial machine.

Third and lastly, owing to the restricted nature of slip near such constrained boundaries, the slip patterns that form in these experiments may not be representative of polyslip, in general. As this is the regime wherein crystal plasticity models have been shown to perform well, further analysis may be required to ascertain the applicability of these models for the bicrystal problem itself.

In future work, it would be necessary to investigate these potential sensitivities much more fully before making general conclusions on the ability of standard crystal plasticity models to capture misorientation inhomogeneities. In the absence of said sensitivities, however, it may still be prudent to consider non-local or gradient crystal plasticity formulations which incorporate fully the rotational degree of freedom and its respective 
force system to resolve the heterogeneous dislocation microstructures observed to develop.

\subsection{Scaling hypothesis}

Next we consider two organizing principles for the diverse data sets that were generated for the different parameters in Chapter 4 . These principles allow us to describe the parameters with a simple function: a scaling hypothesis, and to predict the interrelationship between the parameters: similitude. Probability distributions of misorientation angle, $\theta$ and boundary spacings, $D$ were calculated from TEM measurements of each dislocation boundary type GNB or IDB. The probability that a given angle can be found between $\theta$ and $\Delta \theta$ is defined as $p\left(\theta, \theta_{a v}\right)$. The scaling hypothesis that is applied is that $p\left(\theta, \theta_{a v}\right)$ is a simple function of the average angle $\theta_{\mathrm{av}}$. Thus

$$
\mathrm{p}\left(\theta, \theta_{\mathrm{av}}\right)=\theta_{\mathrm{av}}{ }^{-1} \mathrm{f}\left(\theta / \theta_{\mathrm{av}}\right)
$$

For the boundary spacings $D, D_{a v}$ replaces $\theta, \theta_{a v}$ in the eq. 5.1.

In the former it has been shown previously that the distributions of IDB misorientation angles as a function of strain, material, temperature and deformation conditions can be described by a single universal function when each of the distributions is scaled by its average angle Hughes et al., 1997 and 1998. At small to medium strain the distributions of GNB misorientation angles also exhibit scaling, although the data collapse is not as good as for the IDBs. In contrast, if all of the boundary types are grouped together into one distribution for a given condition, then scaling does not occur. Furthermore it was shown (Godfrey and Hughes, 2000) that a similar scaling hypothesis can be applied to the distribution of GNB spacing. The observed scaling laws are augmented by the observation of a power law relationship between the average misorientation and strain $\theta_{\mathrm{av}}=\mathrm{K} \varepsilon^{\mathrm{n}}$, in which $\mathrm{K}$ is a material and defomation mode dependent constant and $\mathrm{n}$ is a number approximately equal to 0.4 for IDBs and 0.8 for GNBs.

The scaling hypothesis has been shown to apply for a large variety of polycrystalline metals and alloys. The present data for single crystals and bicrystals have shown that both the misorientation angle distributions and the GNB spacing distributions fit into the common framework (e.g. Figure 57). This result shows that the same scaling hypothesis applies to both polycrystals and single crystals. 


\subsection{Modeling Nucleation of Recrystallization.}

Mesoscale recrystallization simulations have indicated that nucleation of strain-free grains is the critical process governing recrystallization dynamics and final grain structure. However, nucleation is an input parameter in grain-scale recrystallization models. Therefore, our mesoscale modeling effort has focused on determining how strain-free nuclei form from the subgrain network. Nucleation of strain-free grains has been proposed to occur when certain "special" subgrains posses boundary energy and/or mobility which favors their growth at the expense of their neighbors. These subgrains then grow beyond some critical size and become grain nuclei. Our theoretical analysis and simulations show that subgrains with boundary energies lower than their neighbors and/or mobilities higher than their neighbors can grow abnormally. Therefore, we must determine whether appropriately advantaged subgrains (i.e. pre-nuclei) exist in real subgrain structures.

A 3-D subgrain structure model was developed using a standard grain growth algorithm. This algorithm yields a geometrically and topologically correct equiaxed subgrain array. Orientations are assigned to subgrains at random with a designated mean misorientation based on the experimental measurements. A Monte Carlo algorithm is used to adjust subgrain orientations at random; reorientations are accepted if they move the structure toward the experimental misorientation distribution. This process was done separately for blocks of cell structures with IDBs. Then long, straight geometrically necessary boundaries (GNBs) are added as planar boundaries to join the cell blocks consistent with the GNB misorientation distribution. Using this method, we have generated realistic microstructures in 3-D which simulate the orientational parameters determined from experiments (Figure 5-8)(Miodownik, Godfrey, Holm and Hughes; 1999).

The Read-Shockley (1950) model for grain boundary energy as a function of misorientation has been implemented in this subgrain model as well as a similar function for grain boundary mobility as a function of misorientation. to perform simulations in search of abnormal subgrain growth as a nucleation mechanism in these realistic structures. This information will feed the mesoscale and continuum recrystallization models.

In parallel to the simulation of realistic structures, simulation of abnormal grain growth in dislocation cell networks (IDB structure) has been investigated (Figure 5-9). The conditions under which such an event may occur have been investigated using a Monte Carlo Potts model. Two sets of simulations were carried out to investigate the effect of outliers in the misorientation distribution function. The initial configurations of a simple 3D dislocation cell microstructures were constructed using a technique outlined above and are statistically equivalent to experimentally measured $\mathrm{IDB}$ structures. Outliers were included by seeding a distribution of $\mathrm{IDB}$ cells, with average misorientation of 1 degree, 
with a few cells with misorientations of 5 degrees. In the first set of simulations, the boundary energies were allocated using the Read-Shockley function with the maxima occurring at 15 degrees. The results of the simulations showed that even when the boundaries of the outlier grains were given mobilities 10 times greater than the IDB cells, they did not abnormally grow. In fact higher mobilities caused them to be removed from the distribution more quickly, and this makes sense in view of the high energy of their boundaries. The effect of size advantage was not investigated. In a second set of simulations the boundaries of the cells were assumed to be in a non-equilibrium state and thus have of energies twice as large as that given by the Read-Shockley function. The simulation allowed recovery of boundaries to take place, the effect of which was to lower boundary energy and increase their mobility. It was found that under conditions in which high-misorientation boundaries recovered quicker than low-misorientation boundaries, abnormal grain growth occurred. A fuller investigation of this phenomenon is ongoing work. 


\section{Continuum Level}

\subsection{Spatial and Rotational Gradient Continuum Level Material Constitutive Model}

A crystal plasticity model was developed which incorporated a natural length scale (see Bammann, 2000) for a more detailed treatment of this model). The length scale is based on a spatial gradient that arises from the presence of dislocations, and comes from a combination of kinematics and compatibility theory. In the following the development of kinematic theory is first traced to identify the appropriate state variables that inherently have length scales. Next compatibility is revisited to bring in a length scale that has significance within both continuum theory and our understanding of defect structures. It is anticipated that more than one than length scale will be necessary to represent real microstructures and their properties. As a first priority it was shown in chapter 3 that local rotations, as revealed by the misorientation angles across dislocation boundaries, were important to describe both the deformation structure and the driving force for recrystallization. The next sections demonstrate that a rotation based length scale can be derived from the rotations in continuum theory.

\subsubsection{Kinematics}

The kinematics of the model was introduced independently by Bilby, 1956, Kroner, 1958 and Lee and Liu, 1967. In their approach the deformation gradient $\mathbf{F}$, is multiplicatively decomposed into elastic and plastic parts.

$$
\mathbf{F}=\mathrm{F}_{\mathrm{e}} \mathrm{F}_{\mathrm{p}}
$$

The intermediate configuration is then reached by unloading elastically through $\mathrm{F}_{\mathrm{e}}^{-1}$. Figure 6.1 shows the different configurations as well as the symbol modifications used to distinguish the different configurations in the equations. The decomposition of $\mathbf{F}$ can be modified through a polar decomposition of the elastic deformation gradient as,

$$
\mathbf{F}_{\mathrm{e}}=\mathrm{V}_{\mathrm{e}} \mathbf{R}_{\mathrm{e}}
$$

in which $V_{e}$ is the elastic stretch and $R_{e}$ is the rotation(that includes both the elastic and rigid components. Then the deformation gradient in (0.1) becomes, 


$$
\mathbf{F}=\mathrm{V}_{\mathrm{e}} \mathbf{R}_{\mathrm{e}} \mathbf{F}_{\mathrm{p}}
$$

The velocity gradient $\mathbf{l}$, is then given as the sum of elastic, rotational and plastic parts as,

$$
\begin{aligned}
\mathbf{l} & =\dot{\mathbf{F}} \mathbf{F}^{-1} \\
& =\dot{\mathbf{V}} \mathbf{V}_{e}^{-1}+\mathbf{V}_{e} \dot{\mathbf{R}}_{e} R_{e}^{\top} \mathbf{V}_{e}^{-1}+V_{e} R_{e} \dot{F}_{p} F_{p}^{-1} R_{e}^{\top} \mathbf{V}_{e}^{-1} \\
& =\mathrm{I}_{e}+V_{e} \bar{\Omega}_{e} V_{e}^{-1}+V_{e} R_{e} \tilde{L}_{p} R_{e}^{\top} \mathbf{V}_{e}^{-1} \\
& =\mathrm{l}_{e}+\omega_{e}+\mathbf{l}_{p}
\end{aligned}
$$

While $\mathbf{l}$ and $\mathbf{l}_{e}$ are naturally defined with respect to the current configuration, the elastic spin, $\omega_{e}$ and plastic velocity gradient, $l_{p}$ are respectively $\bar{\Omega}_{e}=\dot{\mathbf{R}}_{e} \mathbf{R}_{e}^{\top}$ and $\tilde{\mathbf{L}}_{p}=\dot{\mathbf{F}}_{\mathrm{p}} \mathbf{F}_{p}^{-1}$, mapped forward to the current configuration. Similarly, the velocity gradient in equation (0.4) can be mapped back to the natural configuration, where the decomposition now takes the form,

$$
\begin{aligned}
\tilde{\mathbf{L}} & =\mathbf{V}_{e}^{-1} \dot{\mathbf{V}}_{e}+\dot{\mathbf{R}}_{e} \mathbf{R}_{e}^{\top}+\mathbf{R}_{e} \dot{\mathbf{F}}_{\mathrm{p}} \mathbf{F}_{p}^{-1} \mathbf{R}_{e}^{\top} \\
& =\tilde{\mathbf{L}}_{e}+\tilde{\Omega}_{e}+\tilde{\mathbf{L}}_{p}
\end{aligned}
$$

The symmetric and skew-symmetric parts of $\tilde{\mathbf{L}}$, respectively, are referred to as the rate of deformation (or stretching) tensor, $\tilde{\mathbf{D}}$, and the vorticity (or spin) tensor, $\tilde{\mathbf{W}}$. Similarly, the velocity gradient tensors $\tilde{\mathbf{L}}_{e}$ and $\tilde{\mathbf{L}}_{\mathrm{p}}$ may be additively decomposed into symmetric and skew symmetric parts as

$$
\begin{aligned}
& \tilde{L}_{e}=\tilde{D}_{e}+\tilde{W}_{e}=\tilde{D}_{e}+\tilde{\Omega}_{e}+\dot{V}_{e} V_{e}^{-1} \\
& \tilde{L}_{p}=\tilde{D}_{p}+\tilde{W}_{p}
\end{aligned}
$$

$\tilde{\Omega}_{e}$ represents the spin of the lattice, while $\tilde{W}_{p}$ represents the spin of a material line through the lattice, and for small elastic deformations $\dot{V}_{e} V_{e}^{-1}$ can be shown to be negligible.. Because of the decomposition of the deformation gradient in equation (0.3), the Lagrange strain in the reference configuration naturally decomposes as the sum of elastic and plastic parts. The total and plastic strains with respect to the reference configuration are therefore of the form, 


$$
\begin{aligned}
& E=\frac{1}{2}\left(\mathbf{F}^{\top} \mathbf{F}-1\right) \\
& E_{p}=\frac{1}{2}\left(\mathbf{F}_{p}^{\top} \mathbf{F}_{p}-1\right)
\end{aligned}
$$

The elastic strain is mapped backwards from the intermediate configuration as,

$$
\begin{aligned}
\mathbf{E}_{e} & =\frac{1}{2} \mathbf{F}_{\mathrm{p}}^{\top} \mathbf{R}_{\mathrm{e}}^{\top}\left(\mathrm{V}_{\mathrm{e}}^{\top} \mathbf{V}_{\mathrm{e}}-1\right) \mathbf{R}_{\mathrm{e}} \mathbf{F}_{\mathrm{p}}=\mathrm{F}_{\mathrm{p}}^{\top} \mathbf{R}_{e}^{\top} \tilde{\mathrm{E}}_{\mathrm{e}} \mathbf{R}_{\mathrm{e}} \mathbf{F}_{\mathrm{p}} \\
& =\mathrm{E}-\mathrm{E}_{\mathrm{p}}
\end{aligned}
$$

Similarly, strains can be defined in terms of each of the components of the deformation gradient and mapped to any configuration. In each configuration the total strain decomposes additively into elastic and plastic strains. In particular, with respect to the natural configuration,

$$
\begin{aligned}
& \tilde{\mathbf{E}}=\mathbf{F}_{\mathrm{p}}^{-\top} \mathbf{R}_{\mathrm{e}} \mathbf{E} \mathbf{R}_{\mathrm{e}}^{\top} \mathbf{F}_{\mathrm{p}}^{-1}=\frac{1}{2} \mathbf{F}_{\mathrm{p}}^{-\top} \mathbf{R}_{\mathrm{e}}\left(\mathbf{F}^{\top} \mathbf{F}-1\right) \mathbf{R}_{\mathrm{e}}^{\top} \mathbf{F}_{\mathrm{p}}^{-1} \\
& \tilde{\mathbf{E}}_{\mathrm{p}}=\mathbf{F}_{\mathrm{p}}^{-\mathrm{T}} \mathbf{E}_{\mathrm{p}} \mathbf{F}_{\mathrm{p}}^{-1}=\frac{1}{2}\left(1-\mathbf{F}_{\mathrm{p}}^{-\top} \mathbf{F}_{\mathrm{p}}^{-1}\right) \\
& \tilde{\mathbf{E}}^{*}=\mathbf{F}_{\mathrm{p}}^{-\top} \mathbf{E}_{\mathrm{e}} \mathbf{F}_{\mathrm{p}}^{-1}=\frac{1}{2}\left(\mathbf{F}_{\mathrm{e}}^{\top} \mathbf{F}_{\mathrm{e}}-\mathbf{1}\right) \\
& \tilde{\mathbf{E}}=\tilde{\mathbf{E}}_{\mathrm{e}}+\tilde{\mathbf{E}}_{\mathrm{p}}
\end{aligned}
$$

The material time derivatives of the various strain measures may be expressed in terms of these velocity gradients as well. The total Lagrangian strain rate is related in the usual way to the rate of deformation tensor as

$$
\dot{\mathrm{E}}=\mathrm{F}^{\top} \mathrm{DF}
$$

Similarly, the material derivative of the plastic strain tensor defined in equation (0.7) is,

$$
\dot{\mathbf{E}}_{\mathrm{p}}=\mathrm{F}_{\mathrm{p}}^{\top} \tilde{\mathbf{D}}_{\mathrm{p}} \mathrm{F}_{\mathrm{p}}
$$

It then follows that,

$$
\dot{\tilde{\mathbf{E}}}_{\mathrm{e}}=\mathrm{D}-\mathrm{D}_{\mathrm{p}}-\tilde{\mathrm{E}}_{e} \tilde{\mathrm{L}}_{\mathrm{p}}-\tilde{\mathbf{L}}_{\mathrm{p}}^{\top} \tilde{\mathbf{E}}_{e}
$$

These are the kinematics necessary to describe the crystal plasticity model. Further details are available in reference [1]. 


\subsubsection{Review of Compatibility Consistent with Dislocations}

Before addressing the thermodynamics of the model, it is useful to briefly review compatibility theory as a motivation for one of the internal state variables. The integrability conditions for a compatible deformation require,

$$
\oint_{\partial \mathcal{B}} \mathbf{d x}=\oint_{\partial \mathcal{B}_{O}} \mathbf{F d X}=\mathbf{0}
$$

where, $\mathbf{d X}$ is an infinitesimal line segment in the reference configuration, $\mathbf{d x}$ is the associated segment in the current configuration and $\partial \mathcal{B}_{O}$ and $\partial \mathcal{B}$ are contours surrounding regions $\mathcal{B}_{O}$ and $\mathcal{B}$ in the reference and current configurations, respectively. Using Stokes theorem, the line integral in equation(0.13) can be converted to an integral over the region $\mathcal{B}_{O}$ with unit normal $\mathbf{N}$, such that

$$
\oint_{\partial \mathcal{B}_{\mathcal{O}}} \mathbf{F d X}=\int_{\mathcal{B}_{\mathcal{O}}}(\nabla \times \mathbf{F}) \mathrm{NdA}=\mathbf{0}
$$

which results in the stronger pointwise requirement,

$$
\nabla \times \mathbf{F}=\mathbf{0}
$$

This ensures compatibility of the current configuration. Since $F_{p}$ is generally not a compatible deformation due to the presence of dislocations, the closure failure $\mathbf{b}$ of the line integral (i.e. the Burgers vector of the dislocation), is defined as

$$
\mathbf{b}=\oint_{\partial \tilde{\mathcal{B}}} \mathbf{d} \tilde{\mathbf{x}}=\oint_{\partial \mathcal{B}_{O}} \mathbf{F}_{\mathrm{p}} \mathbf{d X}=\int_{\mathcal{B}_{O}}\left(\nabla \times \mathbf{F}_{\mathrm{p}}\right) \mathbf{N d A}
$$

where $\mathbf{d} \tilde{\mathbf{X}}$ is an infinitesimal line segment in a region $\tilde{\mathcal{B}}$ of the intermediate configuration. It is natural to define a tensor $\alpha_{p}$ representing this incompatibility as,

$$
\alpha_{p}=\nabla \times F_{p}
$$

Since $F_{p}$ is a two point tensor connecting the reference configuration $\lambda_{0}$ to configuration $\lambda_{1}, \alpha_{p}$ is also a two point tensor connecting the same configurations. Since $\mathbf{F}$ is a compatible deformation field, $\mathrm{F}_{\mathrm{e}}$ must represent an elastic rotation which restores the incompatibility resulting from the deformation $\mathbf{F}_{\mathrm{p}}$. To see this substitute equation (0.1) into equation (0.15) 
which leads to a statement of compatibility for the total deformation $F$ of the form,

$$
\tilde{\alpha}_{p}-\tilde{\alpha}_{e}=0
$$

Where,

$$
\tilde{\alpha}_{p}=F_{p} \alpha_{p}=F_{p}\left(\nabla \times F_{p}\right) \quad ; \quad \tilde{\alpha}_{e}=\alpha_{e} F_{e}^{-1}=\left(\nabla \times F_{e}\right) F_{e}^{-1}
$$

These equations were obtained previously by Teodosiu, 1970, Werne,1976 and recently by Steinmann,1996, while the small strain formulation of these equations was first obtained by Kroner, 1958. Bilby et al., 1956 and Kondo, 1952 using concepts from tensor calculus also obtained the dislocation density tensor. In this approach, the dislocation density tensor is a result of the fact that the Cartan torsion tensor does not vanish and therefore the intermediate configuration is not an Euclidean space. Also, as will be shown in the sequel, Steinmann develops a theory that involves a second order spatial gradient, instead of the first order gradient associated with $\tilde{\alpha}_{e}$. These results can be shown (Bammann, 2000) to have a direct connection to Nye's, 1953, dislocation density tensor and geometrically necessary dislocations. These results were used to solve the elasticity problem of the residual stress field arising from various distributions of dislocations. In terms of an internal state variable theory, $\tilde{\alpha}_{e}$ becomes the source of internal stress. In the following section, a similar state variable is introduced based upon the kinematics of equation (0.3). The curvature, $\tilde{\mathrm{\kappa}}_{\mathrm{e}}$ is defined as

$$
\tilde{\mathbf{K}}_{e}=\left(\nabla \times \mathbf{R}_{e}\right) \mathbf{R}_{e}^{\top}
$$

and introduced as an internal state variable providing the necessary length scale while the elastic strain $\tilde{\mathbf{E}}_{e}$ represents the superposed elastic strain from additional loading of the body.

\subsubsection{Thermodynamics}

In this section an internal state variable model is developed following the thermodynamics proposed by Coleman and Gurtin,1967. This approach was first utilized in conjunction with continuum dislocation theory by Teodosiu 1970 , and has been subsequently used by many authors. The reduced entropy inequality takes the following form with respect to the intermediate configuration (Bammann, 2000). 


$$
-\tilde{\rho} \tilde{\tilde{\psi}}-\tilde{\rho} \eta \dot{\Theta}+\tilde{\boldsymbol{\sigma}} \cdot \tilde{L}^{\top}-\frac{1}{\Theta} \tilde{\mathbf{q}} \cdot \tilde{\nabla} \Theta \geq 0
$$

where, $\tilde{\rho}$ is the material density, $\tilde{\psi}$ is the Helmholtz free energy, $\tilde{\mathbf{q}}$ is the heat flux, $\Theta$ is the temperature, $\eta$, the entropy, $\tilde{\nabla}$ the gradient with respect to intermediate configuration, and $\tilde{\boldsymbol{\sigma}}$ is the Cauchy stress mapped backwards from the current configuration to the intermediate configuration by,

$$
\tilde{\boldsymbol{\sigma}}=\operatorname{det} \mathrm{V}_{\mathrm{e}} \mathrm{F}_{\mathrm{e}}^{-1} \tilde{\boldsymbol{\sigma}} \mathrm{F}_{\mathrm{e}}^{-\top}
$$

Thermodynamic restrictions are obtained by making specific assumptions of the form of the dependence of the kinetic variables upon the kinematic variables. Specifically, assume that the free energy, stress and temperature depend upon the elastic strain defined with respect to the intermediate configuration, the temperature and two internal state variables. Two natural choices are the elastic strain $\tilde{\varepsilon}_{\text {Ss }}$ associated with the density of statistically stored dislocations $\rho_{s s}$, and the curvature $\tilde{\kappa}_{e}$ (as defined in equation (0.20)), resulting from the presence of the geometrically necessary dislocations generated during the plastic slip. The energy of a network of dislocations is proportional to the density of dislocations and therefore it is easy to associate $\tilde{\varepsilon}_{\text {ss }}$ with

$$
\tilde{\varepsilon}_{\mathrm{ss}}=\mathrm{b} \sqrt{\rho_{\mathrm{ss}}}
$$

The free energy therefore takes the form,

$$
\begin{aligned}
& \tilde{\psi}=\tilde{\psi}\left(\tilde{\mathrm{E}}_{e}, \Theta, \varepsilon_{\mathrm{SS}}, \tilde{\mathrm{K}}_{e}\right) \\
& \tilde{\boldsymbol{\sigma}}=\tilde{\boldsymbol{\sigma}}\left(\tilde{\mathrm{E}}_{e}, \Theta, \varepsilon_{\mathrm{SS}}, \tilde{\mathrm{K}}_{e}\right) \\
& \eta=\eta\left(\tilde{\mathrm{E}}_{e}, \Theta, \varepsilon_{\mathrm{SS}}, \tilde{\mathrm{K}}_{e}\right)
\end{aligned}
$$

Substituting into equation (0.21) and using equation (0.12) to relate the velocity gradient to the material derivative of the elastic strain tensor, it follows that

$$
\begin{aligned}
& \left(\tilde{\boldsymbol{\sigma}}-\tilde{\rho} \frac{\partial \tilde{\psi}}{\partial \tilde{\mathrm{E}}_{e}}\right) \cdot \dot{\tilde{E}}_{e}-\tilde{\rho}\left(\eta+\frac{\partial \tilde{\psi}}{\partial \Theta}\right) \dot{\Theta}+\tilde{\boldsymbol{\sigma}} \cdot\left(\tilde{\mathbf{D}}_{p}+\tilde{\mathrm{E}}_{e} \tilde{L}_{p}+\tilde{L}_{p}^{\top} \tilde{E}_{e}\right) \\
& -\frac{\partial \tilde{\psi}}{\partial \varepsilon_{s S}} \dot{\varepsilon}_{S S}-\frac{\partial \tilde{\psi}}{\partial \tilde{K}_{e}} \cdot \dot{\tilde{\mathbf{K}}}_{e}-\frac{1}{\Theta} \tilde{\boldsymbol{q}} \cdot \tilde{\nabla} \Theta \geq 0
\end{aligned}
$$

Since equation (0.25) must hold for all motions, it follows 


$$
\begin{aligned}
& \tilde{\boldsymbol{\sigma}}=\tilde{\rho} \frac{\partial \tilde{\psi}}{\partial \tilde{\mathbf{E}}_{e}} \\
& \eta=-\frac{\partial \tilde{\psi}}{\partial \Theta} \\
& \tilde{\boldsymbol{\sigma}} \cdot\left(\tilde{\mathbf{D}}_{\mathrm{p}}+\tilde{\mathbf{E}}_{e} \tilde{\mathbf{L}}_{p}+\tilde{\mathbf{L}}_{p}^{\top} \tilde{\mathbf{E}}_{e}\right)-\tilde{\tau} \dot{\varepsilon}_{s s}-\tilde{\chi} \cdot \dot{\tilde{\mathbf{K}}}_{e}-\frac{1}{\Theta} \tilde{\mathbf{q}} \cdot \tilde{\nabla} \Theta \geq 0
\end{aligned}
$$

Where the internal resistances (or stresses) $\tilde{\chi}$ and $\tilde{\tau}$ are defined as,

$$
\begin{gathered}
\tilde{\chi} \equiv \frac{\partial \tilde{\psi}}{\partial \kappa_{e}} \\
\tilde{\tau} \equiv \frac{\partial \tilde{\psi}}{\partial \varepsilon_{\mathrm{SS}}}
\end{gathered}
$$

Consistent with the assumption of small elastic strains, assume a free energy of the form

$$
\begin{aligned}
\tilde{\rho} \tilde{\psi}\left(\tilde{\mathbf{E}}_{e}, \Theta, \tilde{\varepsilon}_{\mathrm{SS}}, \tilde{\mathbf{K}}_{\mathrm{e}}\right)= & \mu(\Theta)\left|\tilde{\mathbf{E}}_{\mathrm{e}}^{\prime}\right|^{2}+\frac{\mathrm{K}(\Theta)}{2} \operatorname{tr}^{2} \tilde{\mathbf{E}}_{\mathrm{e}} \\
& +\mathrm{c}_{\tilde{\tau}} \mu(\Theta) \tilde{\varepsilon}_{\mathrm{SS}}^{2}+\ell_{1} \mu(\Theta)\left|\tilde{\mathbf{K}}_{\mathrm{e}}\right|^{2}+\mathrm{g}(\Theta)
\end{aligned}
$$

in which $\mu(\Theta)$ is the shear modulus, $K(\Theta)$ is the bulk modulus, $g(\Theta)$ is the temperature dependence and $\ell_{1}$ is the length scale. The first two terms result in standard linear elasticity,

$$
\begin{aligned}
& \tilde{\sigma}=2 \mu(\Theta) E_{e}^{\prime} \\
& \operatorname{tr} \tilde{\sigma}=3 K(\Theta) \operatorname{tr} E_{\Theta}^{\prime}
\end{aligned}
$$

While the third and fourth terms result in expression for the internal stresses arising from the statistically stored dislocations and the curvature,

$$
\begin{aligned}
& \tilde{\tau}=\mu(\Theta) \varepsilon_{\text {SS }}=\mu(\Theta) b \sqrt{\rho_{\text {SS }}} \\
& \tilde{\chi}=\ell_{1} \mu(\Theta) b \tilde{\kappa}_{e}
\end{aligned}
$$




\subsection{Crystal Plasticity Model}

It is convenient to couple the concepts of the previously derived internal stresses to a model for crystal plasticity. Since the stress $\tilde{\chi}$ is proportional to a gradient term, a natural length scale is introduced into the model. For crystal slip along planes defined by unit normal $\tilde{\mathbf{m}}^{(i)}$ and slip direction $\tilde{\mathbf{s}}^{(i)}$, the plastic stretch and spin take the form

$$
\begin{aligned}
& \tilde{\mathbf{D}}_{\mathrm{p}}=\sum_{\mathrm{i}} \dot{\gamma}^{(i)}\left(\tilde{\mathbf{m}}^{(i)} \otimes \tilde{\mathbf{s}}^{(i)}+\tilde{\mathbf{s}}^{(i)} \otimes \tilde{\mathbf{m}}^{(i)}\right) \\
& \tilde{\mathbf{W}}_{\mathrm{p}}=\sum_{\mathrm{i}} \dot{\gamma}^{(i)}\left(\tilde{\mathbf{m}}^{(i)} \otimes \tilde{\mathbf{s}}^{(i)}-\tilde{\mathbf{s}}^{(i)} \otimes \tilde{\mathbf{m}}^{(i)}\right)
\end{aligned}
$$

Now assume that the function $\dot{\gamma}^{(i)}$ is of the form,

$$
\dot{\gamma}^{(i)}=\dot{\gamma}_{0}^{(i)}\left(\frac{(\tilde{\boldsymbol{\sigma}}-\tilde{\chi}) \cdot \tilde{\mathbf{m}}^{(i)} \otimes \tilde{\mathbf{s}}^{(i)}-\tilde{\tau}}{\sigma_{0}}\right)^{\frac{1}{m}}
$$

To determine the internal stresses in equation (0.32), it is necessary to specify the evolution of $\rho_{\text {ss }}$. The statistically stored $\left(\rho_{\text {ss }}\right)$ dislocations are stored proportional to their mean free path, which initially, is inversely proportional to the square root of the density of statistically stored dislocations. As the deformation continues to large strains and therefore rotations, the misorientation increases and the mean free path is controlled by the smaller cell size. Assuming that these are parallel processes, the mean free path $\lambda$ is the inverse of the sum of the inverse associated with each process, hence

$$
\lambda=\left[\frac{1}{\lambda_{\mathrm{ss}}}+\frac{1}{\lambda_{\mathrm{gn}}}\right]^{-1} .
$$

Now assume that each mean free path is inversely proportional to square root of the density, respectively,

$$
\lambda_{\mathrm{ss}}=\frac{1}{\sqrt{\rho_{\mathrm{ss}}}} ; \lambda_{\mathrm{gn}}=\frac{1}{\sqrt{\rho_{\mathrm{gn}}}}
$$

Where, 


$$
\rho_{\mathrm{gn}} \equiv\left(\tilde{\mathbf{K}}_{\mathrm{e}} \tilde{\mathbf{m}}\right) \cdot \tilde{\mathbf{s}}
$$

Then, it follows that

$$
\frac{c_{1}}{\lambda}=c_{1}\left(\sqrt{\rho_{s s}}+\sqrt{\rho_{\mathrm{gn}}}\right)
$$

And, the evolution for the statistically stored dislocations becomes

$$
\begin{aligned}
\dot{\rho}_{\mathrm{ss}} & =\frac{c_{1}}{\lambda} \dot{\gamma}^{(i)}-c_{2} \rho_{\mathrm{ss}} \dot{\gamma}^{(i)} \\
& =c_{1}\left(\sqrt{\rho_{\mathrm{GN}}}+\sqrt{\rho_{\mathrm{ss}}}\right) \dot{\gamma}^{(i)}-c_{2} \rho_{\mathrm{ss}} \dot{\gamma}^{(i)}
\end{aligned}
$$

And therefore the length scale enters the model through the evolution of hardening at large strains. A similar assumption has been made by Beaudoin and Acharya, $2000=$, to obtain a better description of sage IV hardening. To determine the stress $\tilde{\chi}$, it is necessary to recall the relationship of $\mathbf{R}_{e}$ in the kinematics. Using equations (0.3) and (0.5) it follows that

$$
\tilde{\omega}_{e}=\dot{\mathbf{R}}_{e} \mathbf{R}_{e}^{\top}=\tilde{\mathbf{W}}-\tilde{\mathbf{W}}_{\mathrm{e}}-\tilde{\mathbf{W}}_{\mathrm{p}}
$$

Therefore, $\mathbf{R}_{e}$ can be calculated by modifying equation ( 0.38$)$

$$
\dot{\mathbf{R}}_{e}=\left(\tilde{\mathbf{W}}-\tilde{\mathbf{W}}_{\mathrm{e}}-\tilde{\mathbf{W}}_{\mathrm{p}}\right) \mathbf{R}_{\mathrm{e}}^{\top}
$$

and the curl of $R_{e}$ can be determined to specify $\tilde{\chi}$ from equation (0.30). In summary, (see Bammann, 2000 for more details), the crystal plasticity model reduces to the following. First the standard assumption of linear elasticity, (this is easily extended to the more natural orthotropic elasticity associated with a crystal, but has been neglected here for simplicity)

$$
\stackrel{\boldsymbol{\sigma}}{=}=2 \mu(\theta)\left(\tilde{\mathbf{D}}^{\prime}-\tilde{\mathbf{D}}_{\mathbf{p}}\right)
$$

The standard equations representing the slip and spin of the crystal, 


$$
\begin{aligned}
& \tilde{\mathbf{D}}_{p}=\sum_{i} \dot{\gamma}^{(i)}\left(\tilde{\mathbf{m}}^{(i)} \otimes \tilde{\mathbf{s}}^{(i)}+\tilde{\mathbf{s}}^{(i)} \otimes \tilde{\mathbf{m}}^{(i)}\right) \\
& \tilde{\mathbf{W}}_{\mathrm{p}}=\sum_{\mathrm{i}} \dot{\gamma}^{(i)}\left(\tilde{\mathbf{m}}^{(i)} \otimes \tilde{\mathbf{s}}^{(i)}-\tilde{\mathbf{s}}^{(i)} \otimes \tilde{\mathbf{m}}^{(i)}\right)
\end{aligned}
$$

where the dependence of the plastic slip on the local stress is of the form,

$$
\dot{\gamma}^{(i)}=\dot{\gamma}_{0}^{(i)}\left(\frac{(\tilde{\boldsymbol{\sigma}}-\tilde{\boldsymbol{\chi}}) \cdot \tilde{\mathbf{m}}^{(\mathrm{i})} \otimes \tilde{\mathbf{s}}^{(i)}-\tilde{\tau}}{\sigma_{0}}\right)^{\frac{1}{\mathrm{~m}}} .
$$

Notice that the length scale is naturally embedded in the model through the back stress $\tilde{\chi}$, which will provide a better description of the elasto-plastic transition. The evolution of the variable associated with statistically stored dislocations is reproduced as above,

$$
\dot{\rho}_{\mathrm{SS}}=c_{1} \frac{\sqrt{\rho_{\mathrm{GN}}} \sqrt{\rho_{\mathrm{sS}}} \dot{\gamma}^{(i)}}{\left(\sqrt{\rho_{\mathrm{GN}}}+\sqrt{\rho_{\mathrm{sS}}}\right)}-c_{2} \rho_{\mathrm{ss}} \dot{\gamma}^{(i)}
$$

And the evolution of the curvature is given as above by,

$$
\dot{\mathbf{R}}_{e}=\left(\mathbf{W}-\mathbf{W}_{e}-\mathbf{W}_{p}\right) \mathbf{R}_{e}^{\top}
$$

In summary, utilizing concepts from the continuum theory of dislocations and thermodynamics of internal state variables, a crystal plasticity model has been developed that includes a length scale. The curvature tensor that was introduced as an internal state variable is directly related to geometrically necessary dislocations (see Bammann, 2000 for details). In the model, it results in a "backstress" which should influence the elasto-plastic transition and increases the hardening at large strains. This variable is also critical in numerical simulations that investigate post-bifurcation response of materials, as well as in the prediction of the formation of misoriented cells at large deformation.

\subsection{Phase Field Model of Recrystallization}

the objective of the phase field research included 1)development of new phase-field paradigms associated with the meso-scale modeling of grain 
boundary kinetics; 2) derivation of an energetic framework for modeling dislocation production; 3)linking of an existing Colorado School of Mines (CSM) internal state variable (ISV) model of plastic flow and static recrystallization to the energetically-based model for dislocation production.

Two phase-field paradigms were developed for this program. The first uses a single scalar field to measure the local disorder along with a set of lattice parameters that characterize grain orientation. Only two wells exist in energy space: one for complete order and one for complete disorder. Because the orientation of a grain does not affect the lattice energy, this approach might be viewed as more physically appealing than the standard theory in which the energy function depends explicitly on orientation. In this paradigm, internal energy is sensitive to gradients in the lattice parameters so that rearrangements of a grain orientation are most easily accomplished in transition zones--i.e. grain boundaries--where the regularized lattice exhibits the lowest order. The method, along with an initial numerical implementation, is the subject of Lisk, 1999. The second paradigm is based on a modulated energy function first considered by Morin, Elder, Sutton, and Grant, 1995 to study polymorphous crystallization. The determinant of a single order parameter tensor is equivalent to the scalar disorder field of first model developed. There are energy wells associated with perfect order at prescribed lattice orientations, and there is also a single well representing complete disorder. Low angle grain boundaries are modeled as transitions that do not involve a decrease in order, and boundaries with a degree of disorder correspond to trapped amorphous material. The work of Morin, Elder, Sutton and Grant, 1995 was extended from twist boundaries to general misorientations by using a tensor order parameter instead of a vector field. This work is reported in Lusk, 1999.

An important part of the CSM effort was to put grain boundary kinetics and dislocation evolution into a single modeling paradigm. As a first step in this direction, dislocation content was modeled as a scalar density field, and a phase-field framework was established that tracks the evolution of dislocation density as a grain boundary moves through a plastically deformed region. Gurtin and Lusk, 1999, summarizes this part of the investigation. Attention was then turned to a means of accounting dislocation production in a model based on thermodynamic driving forces. Motivating this was the need to be able to consider grain boundary motion and interaction with dislocations in the same simulation with dislocation production. A modulated energy function was used to accomplish this goal. Moreover, it was found that a macroscopic kinetic equation for dislocation production could be derived from its mesoscopic counterpart. This established links between dislocation production, macroscopic yield, and material hardening parameters. The CSM group had, from a previous research project, created an ISV model that 
coupled plastic deformation to static recrystallization, and so this link enabled the ISV model to be fitted using phase-field parameters. The results of this effort are reported in Grach, Lusk and Bammann, 2000.

Future research in this area should attempt to fit and apply the phase-field and internal state variable models developed in this investigation. Although the models were in place shortly after year two, logistics problems prevented their fitting with the experimental data generated in this project. During year three, the CSM group considered the strengths and weaknesses of phase-field and Q-state Potts model simulations of grain boundary kinetics. One outcome of this effort was a Potts model for grain kinetics that is coupled to a finite element simulator for elastic deformation. The approach can be extended to include plastic deformation but that was not undertaken. A second outcome of the final year of work was a statistical model that, in some ways, is a hybrid between the phase-field and Potts model simulations. The new scheme, referred to as a discrete gradient method, has the same discrete kinematics as the Potts model. However, evolution events are always selected that cause the greatest decrease in local energy, and the probability of acceptance of such events is proportional to the decrease in free energy. This is analogous to the phase-field model, wherein evolution rate is obtained using variational derivatives of the relevant free energy. Work in this area is still ongoing. 


\section{Achievements}

The most significant achievements in our LDRD were:

1) to capture the rotational distribution of the microstructure, which is the key parameter controlling the local and overall driving force for recrystallization;

2) to sequentially incorporate this experimentally captured parameter into a scaling model, a three dimensional microstructure evolution model, and a continuum level constitutive model;

3) to develop a crystal plasticity model which incorporates a physical length scale and additional rotational degrees of freedom.

Specific accomplishments at the continuum level material constitutive law include: 1) At the macro scale, an additional state variable has been added to the rate and temperature dependent BCJ plasticity model (nongradient version) to simulate the stress change during dynamic recrystallization. (This work was done in conjunction with Georgia Tech.) The evolution of the variable depends upon a critical function of the existing state variables, strain rate and temperature. The model compares reasonably well with data on copper compression specimens. 2) The kinematics of finite deformation plasticity have been incorporated into the thermodynamic approach of Gurtin and Fried. This formulation was extended to endow the continuum with an extra rotational degree of freedom by decomposing the deformation gradient into elastic, plastic and rotational parts. The rotational aspects stem from the experimental observations of "universal" scaling for deformation microstructure evolution. This same approach of Gurtin and Fried has been utilized to develop a phase-field theory for use in modeling grain growth and recrystallization from a continuum perspective.

At the meso-scale we have incorporated 3-D dislocation substructures determined by experiment and Monte Carlo modeling into the meso-scale recrystallization model. The mesoscale modeling effort has focused on determining how strain-free nuclei form from the subgrain network. To convert the 2-D data to a 3-D subgrain structure a new Monte Carlo algorithm was developed that adjusts subgrain orientations to reflect the experimentally observed scaling laws for misorientation angles. Using this method, we have generated realistic microstructures in 3-D which match all geometric and orientational parameters determined from experiments. The Read-Shockley model for grain boundary energy as a function of misorientation has been implemented in the subgrain model. Grain boundary mobilities from molecular dynamics simulations were incorporated into subgrain growth simulations. 3-D simulations of grain growth with 
anisotropic grain boundary energy were performed and showed behavior consistent with the thermodynamic analyses.

At the atomistic to grain scale, the deformation characteristics and resulting dislocation structure of the deformed and subsequently recrystallized bicrystals are important to the nucleation study. Bicrystals were grown, deformed, recrystallized and quantitatively characterized with Laue $\mathrm{x}$-ray diffraction, transmission electron microscopy (TEM), and electron backscattered pattern analysis in the scanning electron microscope (SEM). The dislocation structure of compressed [421] bicrystals was observed and measured in the TEM and SEM. This characterization revealed a very layered microstructure with large rotations observed in narrow bands separating matrix regions with much smaller rotations. Subsequently upon heat treating, the banded regions with the highest rotations were observed to be nucleation sites for recrystallization which appears to take place in a two stage process. The strong nucleation potential of the bands appeared to over ride the potential of sites at the grain boundary present in the bicrystal. The relationship between the rotations and the nucleation sites provides criteria for the mesoscale recrystallization model and for the nonlocal continuum model.

\section{Future Work}

This investigation has given firm guidelines for future work combining experimental characterization and modeling with the development of continuum level constitutive models.

A key problem that should be addressed is the evolution of structural parameters related to processing conditions during plastic deformation and simulation of structural evolution by nonlocal continuum modeling. A further step would be to incorporate a continuum level model into a FEM code.

Another line of research should focus on the physics of the nucleation processes taking place during recrystallization of heterogeneous deformation microstructures. This line must combine detailed microstructural studies with atomistic simulations. A further step would be to incorporate physically based nucleation models into 3-D models of the microstructural and textural evolution during recrystallization.

Finally a future goal would be an FEM code, that contains these models, to be used in industrial thermo-mechanical processing of metals linking processing parameters to the properties of a product. 


\section{Appendix A Bicrystal Boundary}

The designation, $\Sigma 29,43.6^{\circ}$ [100], means that the two crystals on either side of the boundary are rotated away from each other by $43.6^{\circ}$ about a [100] crystal axes lying in the boundary plane. This rotation creates a special relationship between the crystals in which some lattice positions in one crystal occupy positions that are coincident with those in the second. The $\Sigma$ designation quantifies the coincidence of the two lattices by giving the ratio between the volume of the unit which defines the coincident lattice sites of the two crystals and the volume of a unit crystal. In this case the ratio is 29 . As another example, twinned crystals, whose lattices share many coincidence sites, have a volume ratio of just 3 .

Our bicrystal boundary geometry was selected to allow atomic imaging of the boundary down [100] with high resolution TEM. Embedded atom calculations, by Steven Foiles, 8726, showed that this boundary plane is stable and should not decompose into facets along along special planes. This result is shown in Figure A-1 with a purposeful boundary step included into the calculations. Boundary facets are a factor which would have influenced any nucleation events occurring along the bicrystal boundary during recrystallization. 


\section{Appendix B Publications Resulting from this LDRD}

D. J. Bammann, "The Continuum Theory of Dislocations: A Natural Length Scale for Crystal Plasticity," submitted to ASME J. Eng. Matls. Tech.

D. J. Bammann, "Simulating Recrystallization Using Internal State Variables," to be submitted to Int. J. Plasticity.

C. C. Battaile and E. A. Holm, "Evolution of 2D Potts Model Grain Microstructures from an Initial Hillert Size Distribution," Grain Growth in Polycrystalline Materials III, eds. H. Weiland, B. L. Adams, and A. D. Rollet (TMS, Warrendale, PA, 1998), 119-124.

G. H. Campbell, S. F. Foiles, H. Huang, D. A. Hughes, W. E. King, D. H. Lassila, D. J. Nikkel, T. Diaz-de-la-Rubia, J. Y. Shu, and V. P. Smyshlyaev, "Multi-Scale Modeling of Polycrystal Plasticity: A Workshop Report," Mater. Sci. Eng. A, 251, (1998), 1-22.

R. D. Doherty, D. A. Hughes, F. J. Humphreys, J. J. Jonas, D. Juul Jensen, M. E. Kassner, W. E. King, T. R. McNelley, H. J. McQueen, and A. D. Rollet, "Aspects of Recrystallization," Mater. Sci. Eng. A, 238/2, (1998), 219-274.

A. Godfrey, and D. A. Hughes, "Scaling of Deformation Induced Dislocation Boundaries," in press Acta Mater., (2000).

A. Godfrey and D. A. Hughes, "Characterization of Dislocation Wall Spacing Distributions," Solidification 1998, ed. S. P. Marsh, et al. (Warrendale: TMS, 1998), 191-199.

G. Grach, M. T. Lusk, and D. J. Bammann, "A Modulated Energy Function for Modeling Dislocation Based Plasticity," : Part1 - Production, to be submitted to Int. J. Plasticity.

M. E. Gurtin, and M. T. Lusk, "Sharp-interface and Phase-field Theories of Recrystallization in the Plane," Physica D, 130, (1999), 133-154.

E. A. Holm and M. Miodownik, "A Mesoscale Model for Strain-Free Grain Nucleation and Recrystallization," Plasticity '99: Constitutive and Damage Modeling of Inelastic Deformation and Phase Transformation (Neat Press, Fulton, MD, 1999), 997-1000.

D. A. Hughes, "Distributions of Low and High Angle Boundaries in Deformed Metals," Recrystallization '99, The Fourth International Conference on 
Recrystallization and Related Phenomena, eds. T. Sakai and H. G. Suzuki (Japan Institute of Metals, 1999), 111-118.

D. A. Hughes, "Grain Subdivision and Moderate to High Angle Boundaries," Proceedings of ReX'96, the Third International Conference on Recrystallization and Related Phenomena, ed. T. R. McNelley (Monterey: Monterey Institute of Advanced Studies, 1997), 171-178.

D. A. Hughes, "The Effect Of Dislocation Microstructures and Grain Subdivision on Crystal Plasticity," Finite Plastic and Viscoplastic Deformation, 6th Proc. of Plasticity and Its Current Applications, ed. A. S. Khan (Fulton: Neat Press, 1997), 253-254.

D. A. Hughes, D. C. Chrzan, Q. Liu, and N. Hansen, "Scaling of misorientation angle distributions," Phys. Rev. Lett., 81, (1998), 4664-4667.

D. A. Hughes, and A. Godfrey, "Microstructure and Texture Development of Compressed [421] Single Crystals and [421]/[13 4 -3] Bicrystals," Plasticity '99 Constitutive Modeling of Inelastic Deformation and Phase

Transformation, ed. A. S. Khan (Fulton, Maryland: Neat Press, 1998), 153156.

H. Jou, and M. Lusk, "Comparison of JMAK Kinetics with a Phase-field Model for Microstructural Evolution Driven by Substructure Energy," Physical Review B, 55 (1997), 8114-8121.

M. T. Lusk, "A Phase-field Paradigm for Recrystallization and Grain Growth," Proceedings of the Royal Society of London, A, 455, (1999), 677-700.

M. T. Lusk, "On a Modulated Energy Function for Simulating Grain Growth and Recrystallization," Submitted to Physical Review, B, (1998).

M. A. Miodownik, "A Monte Carlo Model of Zener Pinning Which Shows f-1 Dependence," Grain Growth in Polycrystalline Materials III, eds. H. Weiland, B. L. Adams, and A. D. Rollet (TMS, Warrendale, PA, 1998), 283-288.

M. A. Miodownik, A. W. Godfrey, E. A. Holm, D. A. Hughes, "On Boundary Misorientation Distribution Functions and How to Incorporate Them into 3D Models of Microstructural Evolution," Acta Mater., 47, (1999), 2661-2668.

M. A. Miodownik, E. A. Holm, A. W. Godfrey, D. A. Hughes, and R. LeSar, "Multiscale Modeling of Recrystallization," Proceedings of the Materials Research Society, Boston, November, 1998, accepted (1999). 
N. Hansen and D. A. Hughes, "Microstructural Evolution and Hardening Parameters," in press Mater. Sci. and Eng., (2000).

A. D. Rollet and E. A. Holm, "Abnormal Grain Growth - The Origin of Recrystallization Nuclei?," Recrystallization 96, ed. T.R. McNelley, Monterey, CA (1996), 31-42.

A. D. Rollett, W. W. Mullins, M. D. Rintoul, and E. A. Holm, "The Link Between Kinetics and Configurational Statistics in Two Dimensional Grain Growth: Comparison of Theory and Simulation," submitted to Proceedings of the International Conference on Grain Growth, (1998). 


\section{Appendix C Presentations Resulting from this LDRD:}

invited: D. J. Bammann, "Introducing a Physical Length Scale into the Continuum," Cal Tech Colloquium, Pasadena, February, (1999).

invited: D. J. Bammann, "Introducing a Physical Length Scale into the Continuum," University of Illinois Colloquium, Urbana-Champaign, January, (1999).

C. C. Battaile and E. A. Holm, "Evolution of 2D Potts Model Grain Microstructures from an Initial Hillert Size Distribution," (Poster) Third International Conference on Grain Growth, Pittsburgh, PA, June, 1998, (given by C. C. Battaile).

C. C. Battaile and E. A. Holm, "Evolution of 2D Potts Model Grain Microstructures from an Initial Hillert Size Distribution," International Conference on Grain Growth, Pittsburgh, (1998).

D. C. Chrzan, "Scaling of Misorientation Angle Distributions in FCC Metals," Theory Institute on Multiple Length-Scale Simulation of Materials Microstructure and Evolution, Argonne, August, (1999).

invited: D. C. Chrzan, "Universal Scaling of Misorientation Angle Distributions," Plasticity '99, Cancun, January, (1999).

D. C. Chrzan, "Toward A Dislocation Based Theory of Cell Formation," 4th US Congress on Computational Mechanics Meeting, San Francisco, August 68, (1997).

A. Godfrey and D. A. Hughes, "Changes in the Dislocation Structure and Grain Subdivision Due to the Presence of a Grain Boundary," Fall 1999 Meeting of the Materials Research Society, Boston, December, (1998).

A. Godfrey and D. A. Hughes, "Characterization of Dislocation Wall Spacing Distributions," ASM/TMS meeting, September, (1997).

invited: G. Grach, M. T. Lusk and D. J. Bammann, "Simulating the Inelasticity of Dislocation Production Using a Modulated Energy Function," Plasticity '99: The Seventh International Symposium on Plasticity and Its Current Applications, Cancun, January, (1999).

invited: Hansen and D. A. Hughes, joint ASME, ASCE, SES summer meeting, Evanston, June 29-Jul 2, (1997). 
invited: E. A. Holm, "Microstructure-Property Relationships," Michigan State University Physics Department Colloquium, Lansing, MI, May, (1999).

invited: E. A. Holm, "Modeling of Microstructure-Property Relationships," 1999 Spring Meeting of the Ohio Section of the APS, Flint, MI, May, (1999).

invited: E. A. Holm, "Modeling of Microstructure-Property Relationships," Gordon Research Conference in Physical Metallurgy, June, (1998).

invited:. E. A. Holm, "Applications of Mesoscale Materials Models at Sandia," Caterpillar Research Group Seminar, September, (1997).

invited: E. A. Holm, "Applications of Mesoscale Materials Models," American Ceramic Society Annual Meeting, Theory and Computational Modeling Symposium, May, (1997).

invited: E. A. Holm, "Boundary Energy and Mobility Effects in Polycrystalline Grain Growth,” ASM/TMS Materials Week '96, Kinetically Determined Particle Shapes Symposium, October, (1996).

invited: E. A. Holm, C. C. Battaile, M. D. Rintoul, H. E. Fang, "Applications of Grain Growth Modeling," Third International Conference on Grain Growth, Pittsburgh, PA, June, (1998).

E. A. Holm, J. W. Cahn, and M. Miodownik, "Boundary motion by discretized mean curvature in the ferromagnetic Ising model," (Poster) TMS Fall Meeting 1998, Evolving Paradigms in Microstructure Evolution Symposium, Rosemont, IL, October, (1998).

invited: E. A. Holm and M. Miodownik, "A Mesoscale Model for Strain-Free Grain Nucleation and Recrystallization," Plasticity '99: 7th International Plasticity Symposium, Cancun, January, (1999), (given by M. Miodownik).

E. A. Holm, N. Zacharopoulos, and D. J. Srolovitz, "Grain Growth in Thermal Gradients," ASM/TMS Materials Week '96, Diffusion and Reaction in Thermal, Electrical Filed and Stress Gradients Symposium, October, (1996).

invited: D. A. Hughes, "Distributions of Low and High Angle Boundaries in Deformed Metals," Recrystallization '99, The Fourth International Conference on Recrystallization and Related Phenomena, Tsukuba City, Japan, July, (1999). 
invited: D. A. Hughes, "Grain Subdivision and Microstructure Evolution During Deformation," Colloquium, Johns Hopkins University, Ann Arbor, May, (1999).

invited: D. A. Hughes, "Grain Subdivision and Microstructure Evolution During Deformation," Colloquium, University of Michigan, April, (1999).

invited: D. A. Hughes, "Dislocation Cell and Boundary Formation," American Physical Society, Atlanta, March, (1999).

invited: D. A. Hughes "Scaling of Dislocation Structure Evolution," NIST Workshop on Work Hardening and Dislocation Patterning in Metals, Pleasanton, June, (1998).

invited: D. A. Hughes, "The Effect Of Dislocation Microstructures and Grain Subdivision on Crystal Plasticity," Finite Plastic and Viscoplastic Deformation, 6th Int. Conf. on Plasticity and Its Current Applications, Juneau, July, (1997).

invited: D. A. Hughes, "Scaling of Deformation Microstructures," Northwestern University, Evanston, June, (1997).

invited: D. A. Hughes, "Cold Deformation and the Nucleation Aspects of Recrystallization," Plasticity '99 Constitutive Modeling of Inelastic Deformation and Phase Transformation, Cancun, January, (1999).

invited: D. A. Hughes, and A. Godfrey, "Microstructure and Texture Development of Compressed [421] Single Crystals and [421]/[13 4 -3] Bicrystals," Plasticity '99 Constitutive Modeling of Inelastic Deformation and Phase Transformation, Cancun, January, (1999).

M. Miodownik, "A Monte Carlo Model of Zener Pinning Which Shows f $\mathrm{f}^{1}$ Dependence," Third International Conference on Grain Growth, Pittsburgh, PA, June, (1998).

M. Miodownik, E. A. Holm, D. A. Hughes, A. W. Godfrey, and R. LeSar, "Multiscale Modeling of Recrystallization," (Poster) Fall 1999 Meeting of the Materials Research Society, Boston, December, (1998).

M. Miodownik, A. D. Rollett, and E. A. Holm, "Boundary motion by curvature in the ferromagnetic Potts model with triple junctions," (Poster) TMS Fall Meeting 1998, Evolving Paradigms in Microstructure Evolution Symposium, Rosemont, IL, October, (1998). 
invited: M. T. Lusk, "Phase-field Modeling of Microstructural Evolution," Student Workshop Seminar, Sandia National Laboratory, Livermore, August, (1999).

invited: M. T. Lusk, "An Alternative Phase-Field Paradigm for Modeling Grain Growth and Recrystallization," Sandia National Laboratory, Albuquerque, June, (1999).

invited: M. T. Lusk, "Modulated Energy Functions and Recrystallization," Materials Research Society, Symposium L: Interaction of Phase and Defect Microstructures in Metallic Alloys, Boston, December, (1998).

invited: M. T. Lusk, "On Phase-Field Paradigms for Grain Growth and Recrystallization," Applied Mechanics Seminar Series, California Institute of Technology, May, (1998).

invited: M. T. Lusk, "A Modulated Energy Function for Grain Growth and Recrystallization," Materials Science Spring Seminar Series, Colorado School of Mines, January, (1998).

invited: M. T. Lusk, "A Phase-Field Paradigm for Grain Growth and Recrystallization," Applied Mechanics Seminar Series, University of Colorado, November, (1997).

invited: M. T. Lusk, "A New Phase-field Model for Grain Growth and Recrystallization," Second Euroconference and International Symposium on Material in Deformation and Fracture, Thessoloniki, Greece, September (1997).

invited: M. T. Lusk, G. Grach, and D. Bammann, "Simulating Inelasticity Using a Modulated Energy Function," Student Workshop Seminar, Sandia National Laboratory, Livermore, August, (1999).

invited: M. T. Lusk and P. Liu, "Accounting for Grain Misorientation via Energy Modulation," Plasticity '99: The Seventh International Symposium on Plasticity and Its Current Applications, Cancun, January, (1999).

invited: M. T. Lusk, and P. Liu, "A Modulated Energy Model for Simulating Grain Boundary Kinetics: Solid State and Granular Media," Society for Engineering Science, Pullman, October, (1998).

invited: A. D. Rollet, W. W. Mullins, and E. A. Holm, "Abnormal Grain Growth - The Origin of Recrystallization Nuclei?," MRS Spring 1997 meeting, Computational Materials Science at the Mesoscale Symposium, April, (1997). 
A. D. Rollett, W. W. Mullins, M. D. Rintoul, and E. A. Holm, "The Link Between Kinetics and Configurational Statistics in Two Dimensional Grain Growth: Comparison of Theory and Simulation," (Poster) Third International Conference on Grain Growth, Pittsburgh, PA, June, (1998), (given by A. D. Rollett). 


\section{REFERENCES}

Bammann, D. J. (2000) to appear J. Eng. Matls. Tech.

Becker, R., Butler, J. F. J., Hu, H. and Lalli, L. (1991) Met. Trans, 22A, 4558.

Beaudoin, A. and A. Acharya, (2000) in press J. Mech. Phys. Solids,

Bilby B. A. and Smith, E. (1956) Proc. Roy. Soc., A 231, 481-505

Cahn, J. W. and Fullman, R. R. (1956) Trans. AIME, 206, 610.

Coleman, B. D. and Gurtin, M. E. (1967) J. Chem. Phys., 47, 597-613.

Dawson, P.R. and Marin, E.B. (1998) Advances in Applied Mechanics, 34, pp. 77-169.

DeHoff, R. T. and Rhines, F. N. (1969) Quantitative Microscopy, McGrawHill, New York.

Godfrey, A. and Hughes, D. A. (1998) In Solidification 1998, Vol. 1 (Eds, Marsh, S. P., Dantzig, J. A., Hofmeister, W., Trivedi, R., Chu, M. G., Lavernia, E. J. and Chun, J. H.) TMS, Warrendale, pp. 191-199.

Godfrey, A. and Hughes, D. A. (2000) Acta mater., in press.

Godfrey, A., Juul-Jensen, D. and Hansen, N. (1998) Acta. Mater., 46, 835-848.

Grach, G, Lusk, M. T. and Bammann, D. J. (2000) to be submitted to Int. J. Plasticity.

Gurtin, M.E. and Lusk, M. T., (1999) Physica D, 130, 133-154.

Hughes, D. A., Chrzan, D. C., Liu, Q. and Hansen, N. (1998) Phys. Rev. Lett., 81, 4664-4667.

Hughes, D. A., Liu, Q., Chrzan, D. C. and Hansen, N. (1997) Acta mater., 45, 105-112.

Kondo, K., in Proc. $2^{\text {nd }}$ Japan Nat. Congr. Appl. Mech., Tokyo, (1952) 41-52

Kroner, E. (1958) Erg. Angew. Math., 5, 1. 
Lee, E. H., and Liu, D. T. (1967) J. Appl. Phys., 38, .

Liu, Q. (1994) J. Appl. Cryst., 27, 755-761.

Liu, Q. and Hansen, N. (1998) Proc. Roy. Soc. Lond., 454, 2555-2591.

Lusk, M.T. (1999) Proc. Roy. Soc. Lond., A, 455, 677-700.

Lusk, M.T. (1999) Submitted to Int. J. of Eng. Sci., B.

Marin, E.B. and Dawson, P. R., (1998) Computational Methods in Applied Mechanics and Eng., 165, pp. 23-41.

Miodownik, M., Godfrey, A. W., Holm, E. A. and Hughes, D. A. (1999) Acta mater., 47, 2661-2668.

Morin, B., Elder, K. R., Sutton, M. and Grant, M. (1995) Phys. Rev. Let. 75, 2156-2159.

Nye, J. F. (1953) Acta Metall., 1, 153-162

Read, W. T. and Shockley, W. (1950) Phys. Rev., 78, 275.

Robinson, S. L., B. C. Odegard, J., Yang, N. Y. C., Hughes, D. A. and Headley, T. (1999) Sandia National Laboratories, Livermore.

Steinmann, P., (1996) Int. J. Eng. Sci., 34, 1717-1736.

Teodosiu, C (1970) in eds. J. A. Simmons, R. de Wit and R. Bullough, U. S.

Nat Bur. Stand. Spec. Publ. 317, 11, 837-876.

Underwood, E. E. (1970) Quantitative Stereology, Addison-Wesley Publishing Co., Reading.

Werne, R. W. (1976) Univ. California Lawrence Livermore Laboratory, Livermore, California UCRL-52002.

Wonsiewicz, B. C. and Chin, G. Y. (1970) Metall. Trans., 1, 57-61. 
a initial

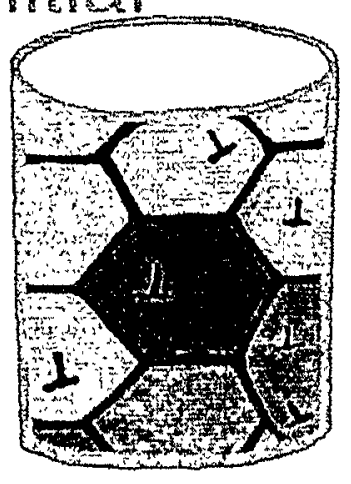

c heat

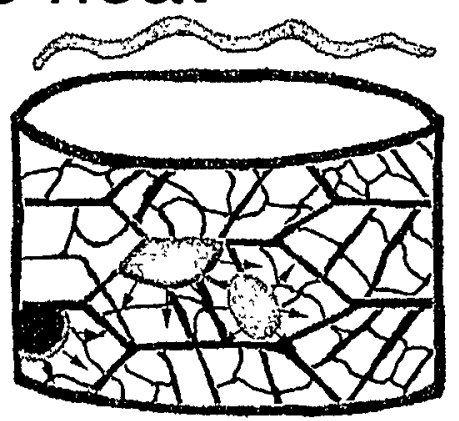

\section{b deform}

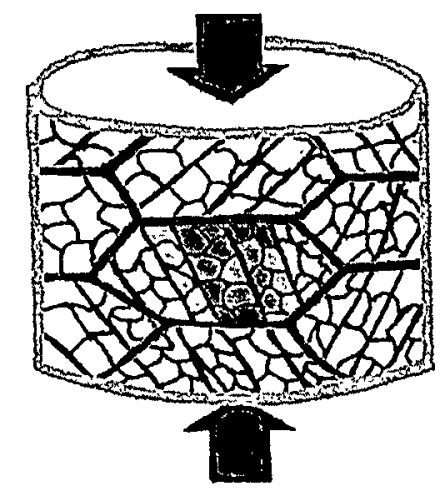

d final

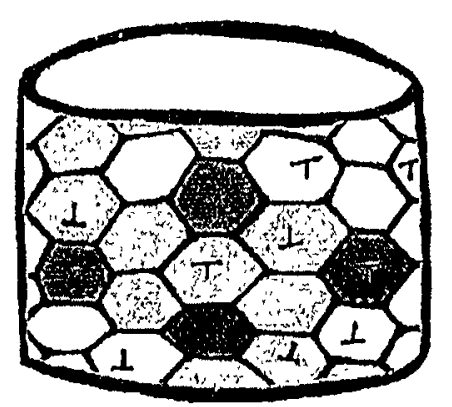

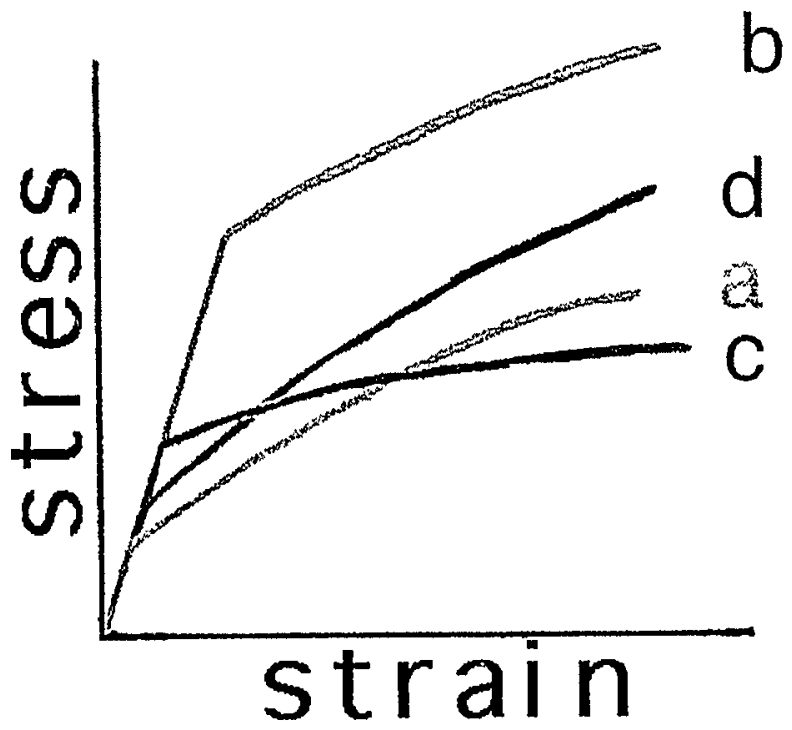

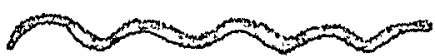

Figure 2-1. Thermo-mechanical processing dramatically changes material properties and microstructure as illustrated by the changes in the stress- strain curve (a) a poly crystal in an initial nearly dislocation free state, (b) following deformation in which dislocation boundaries form, (c) following partial annealing in which some recrystallization nuclei form and (d) after full recrystallization to a new grain size and crystallographic texture. 


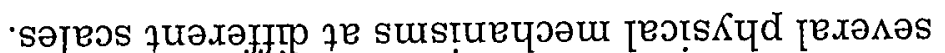

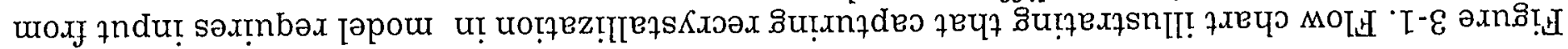

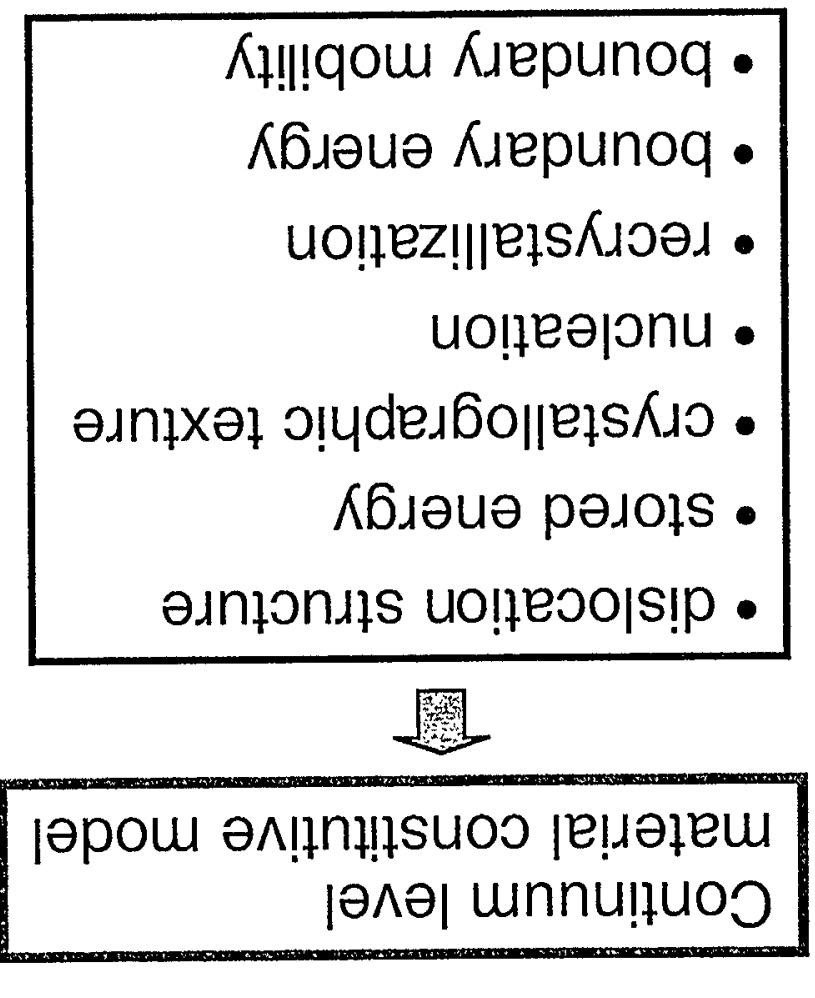




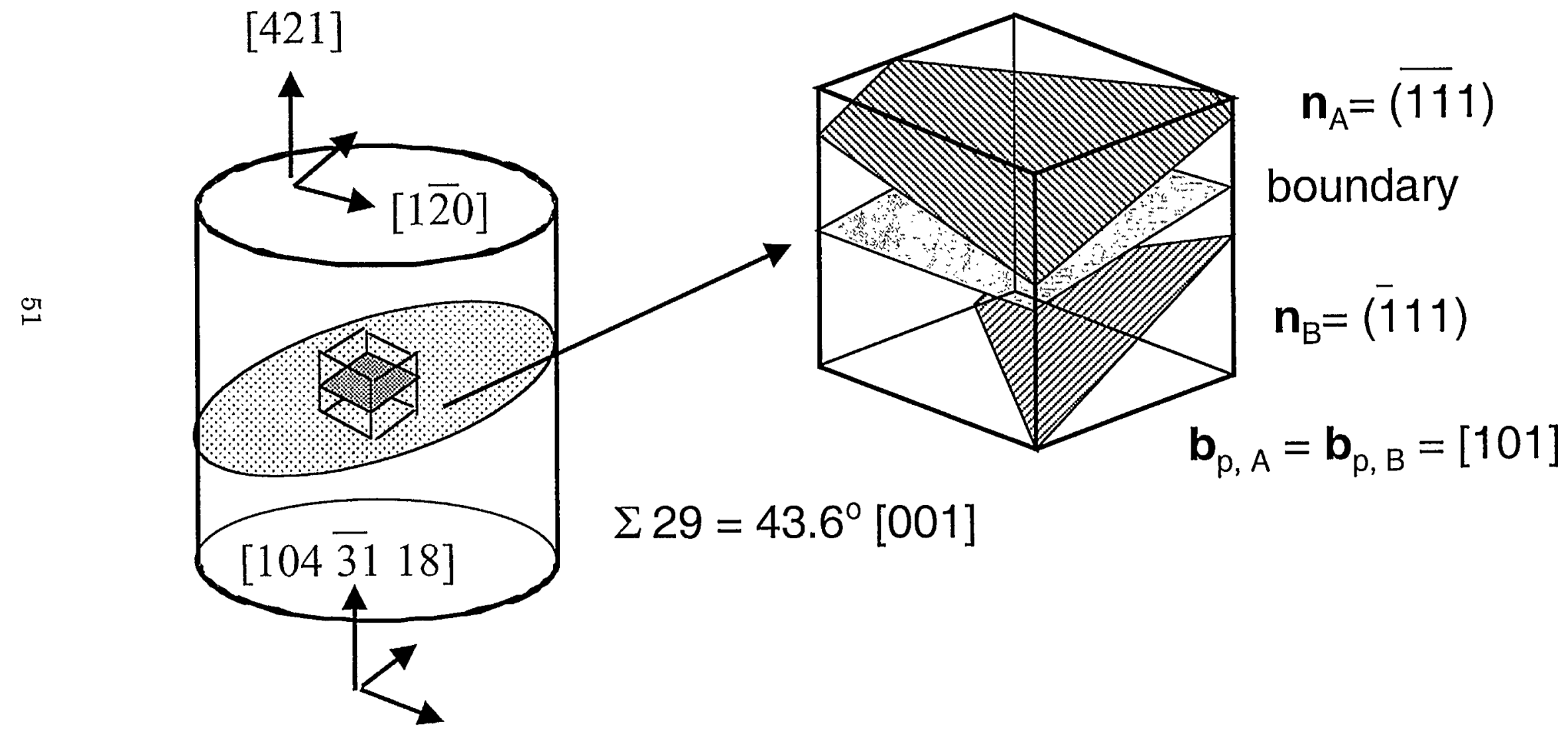

Figure 4-1. Schematic drawing of the bicrystal showing the location of the bicrystal boundary and primary slip planes with respect to the compression sample. The index A is used for [421] and B for the [10431 18] crystals slip plane normal $n$ and Burgers vector $b$. 


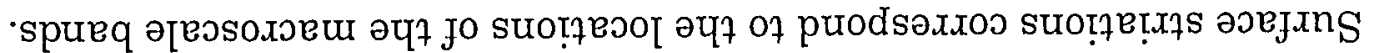

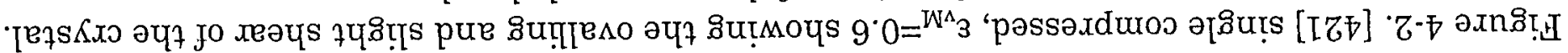
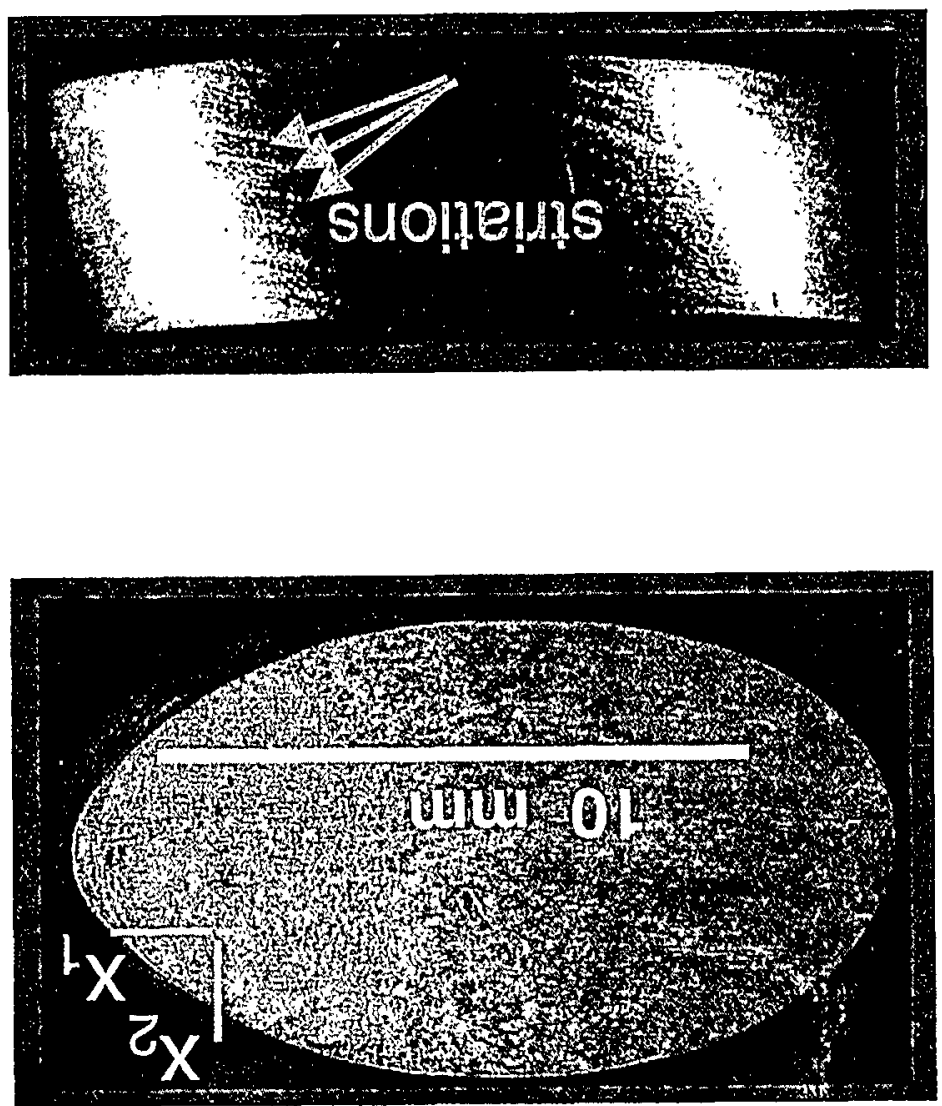

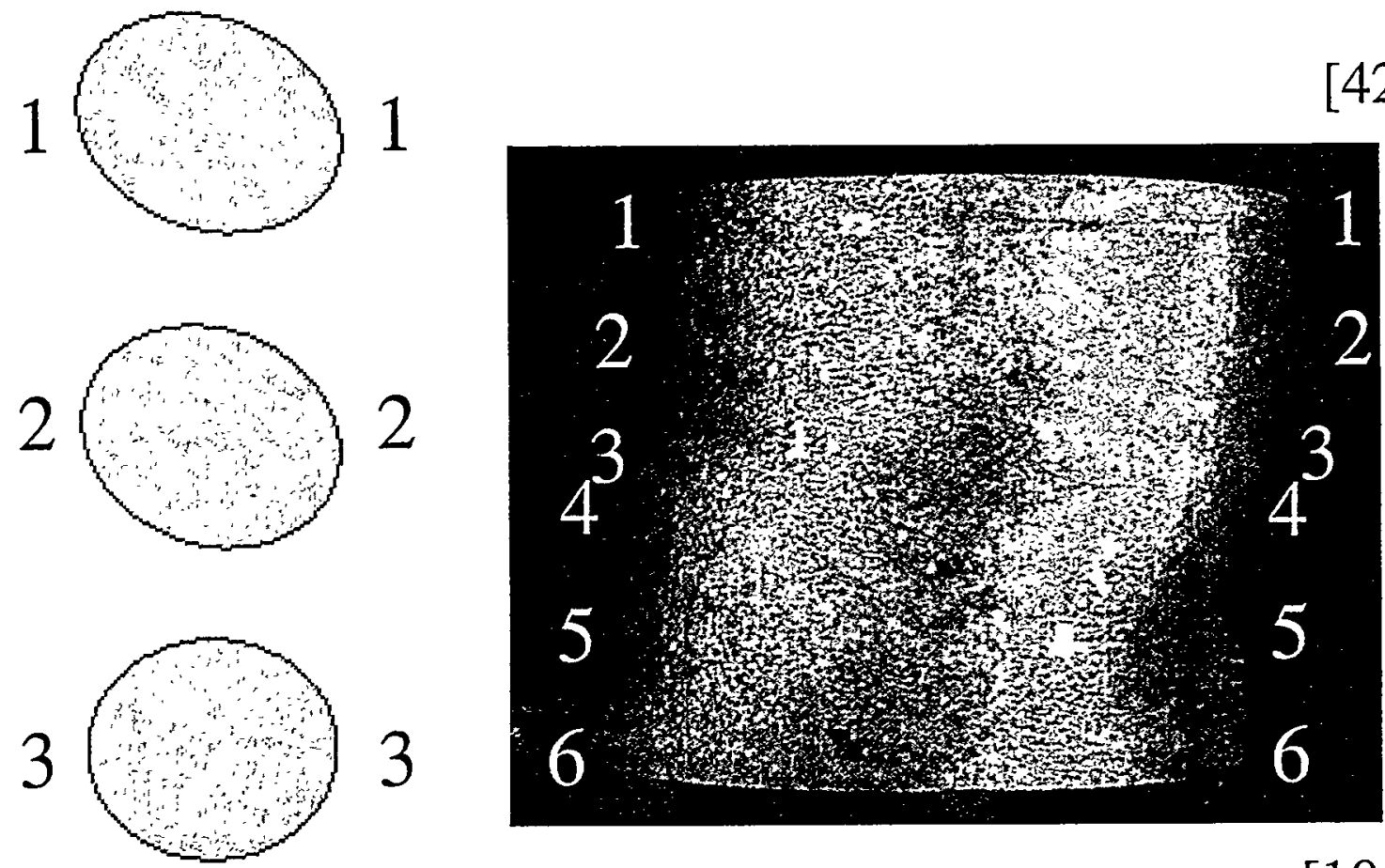

(side view)
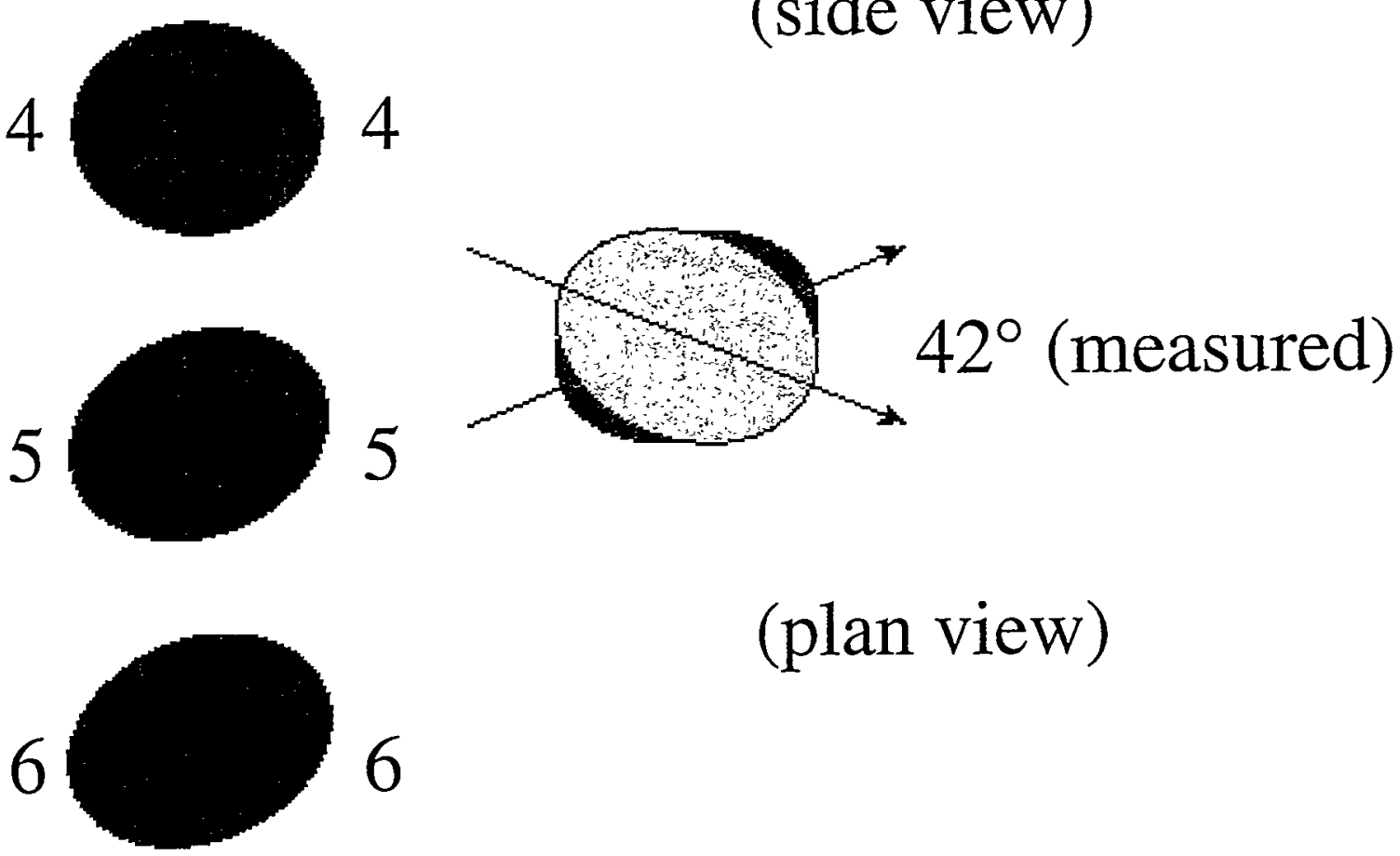

(plan view)

Figure 4-3. Cross sections of the $\varepsilon_{\mathrm{vM}}=0.3$ deformed bicrystal showing ovalling in the primary slip directions. 
a)

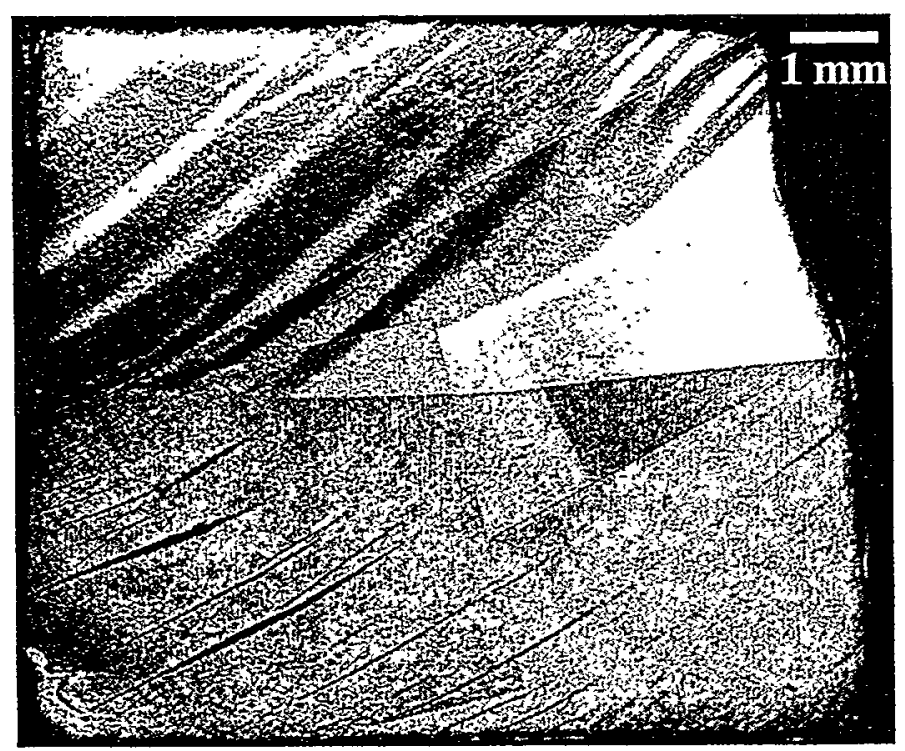

b)

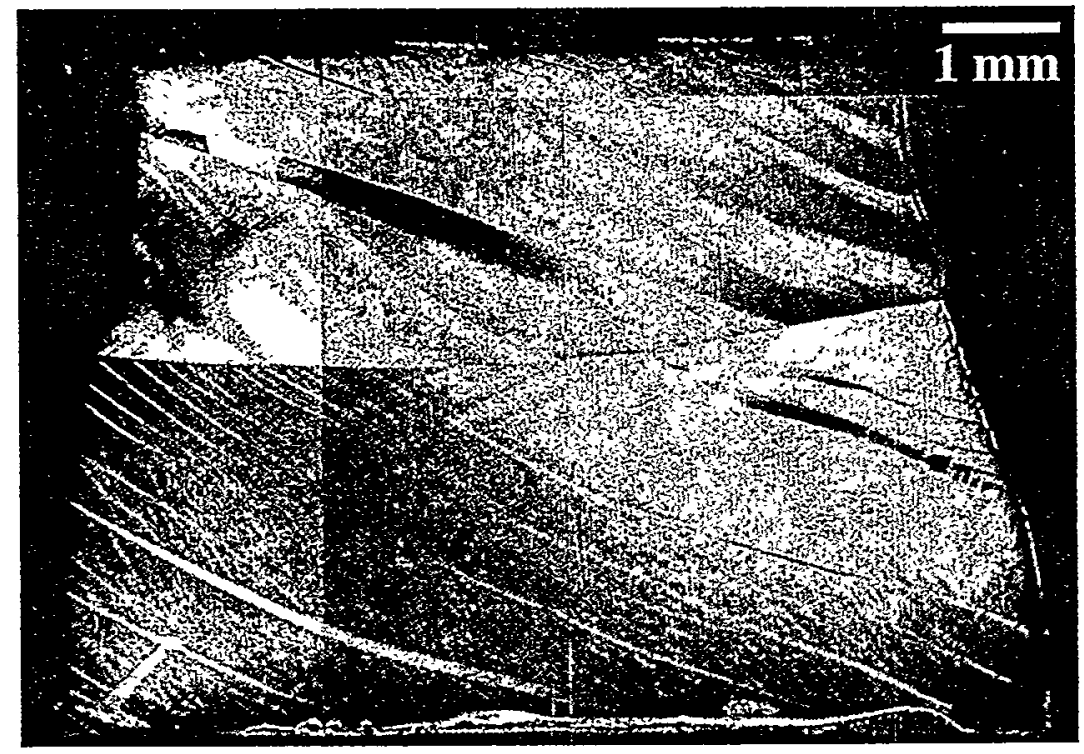

Figure 4-4 Optical microsgxphs of anodized cross-sections of the bicrystal deformed a) $30 \%$ and b) $45 \%$ showing macroscale banding. 


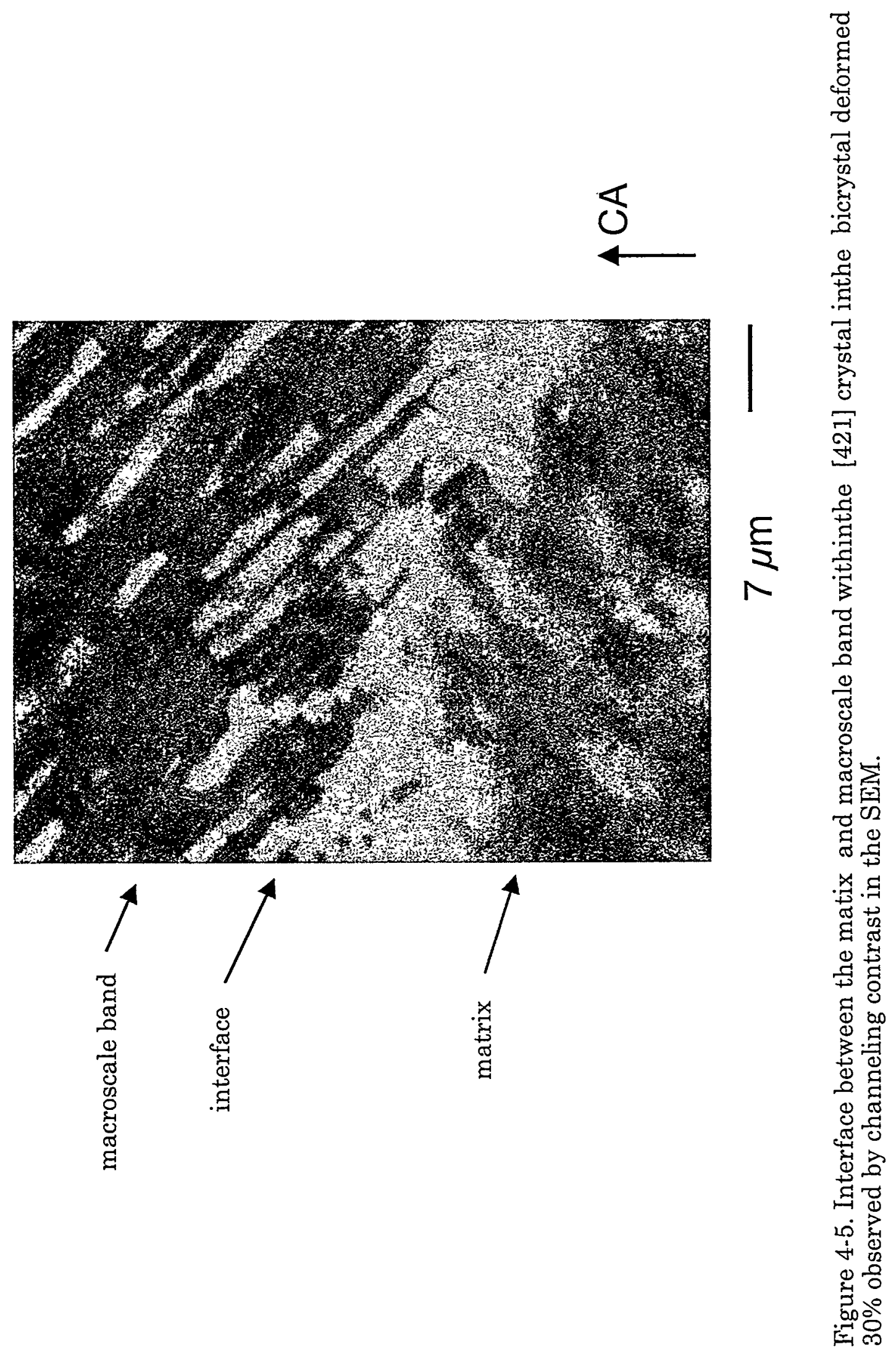




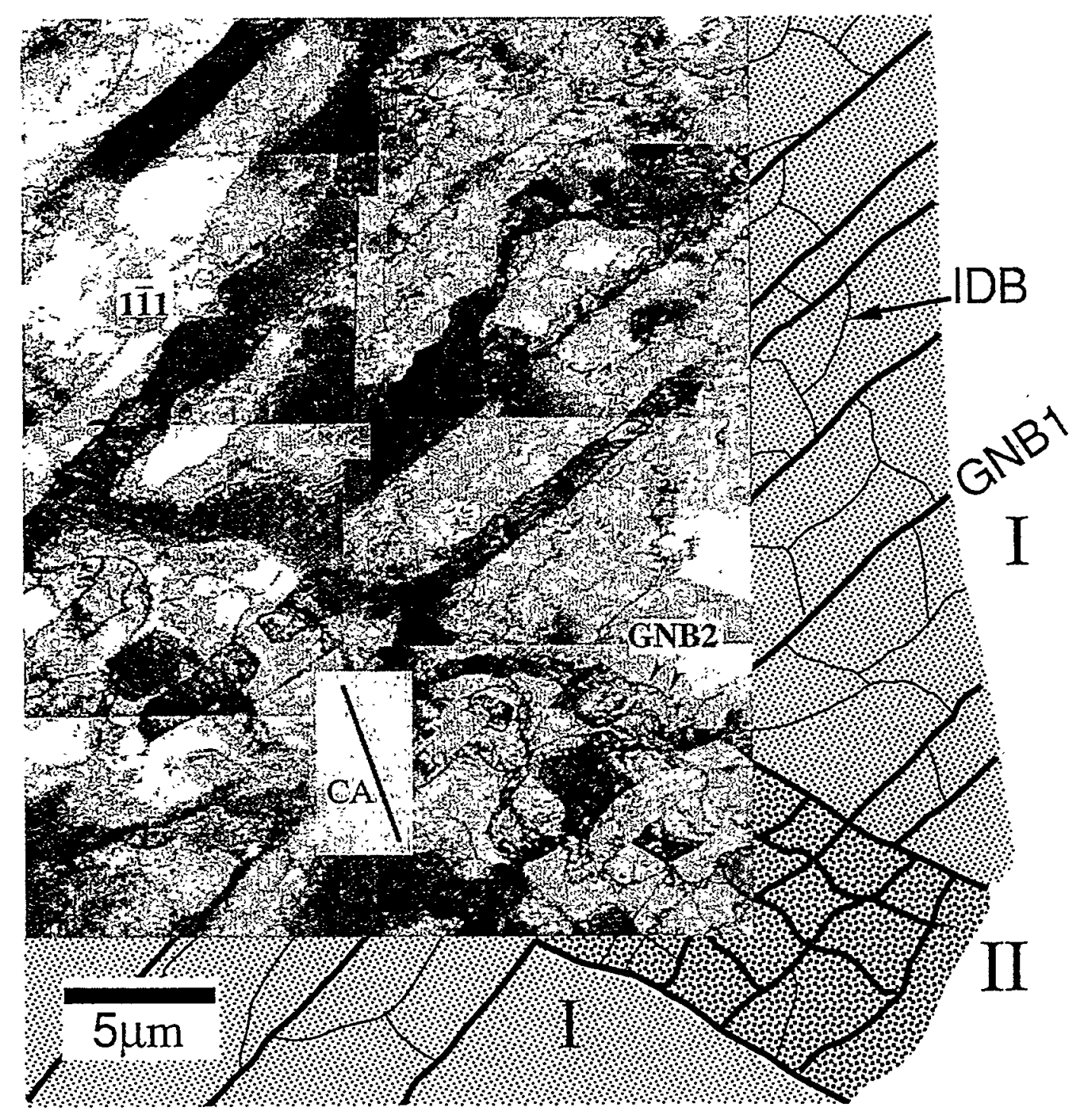

Figure 4-6. TEM micrograph of the matrix (I) and the macroscale bands (II) in the [421] single crystal compressed to $\varepsilon_{\mathrm{vM}}=0.3$. 







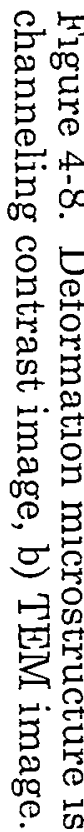

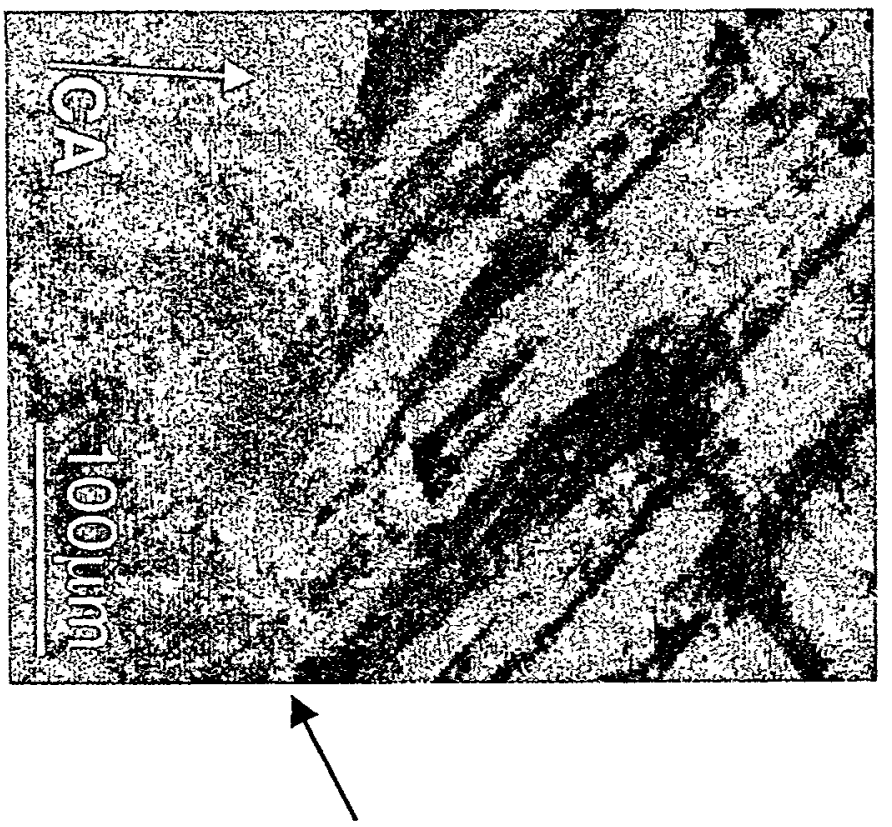

$?$

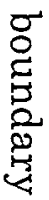

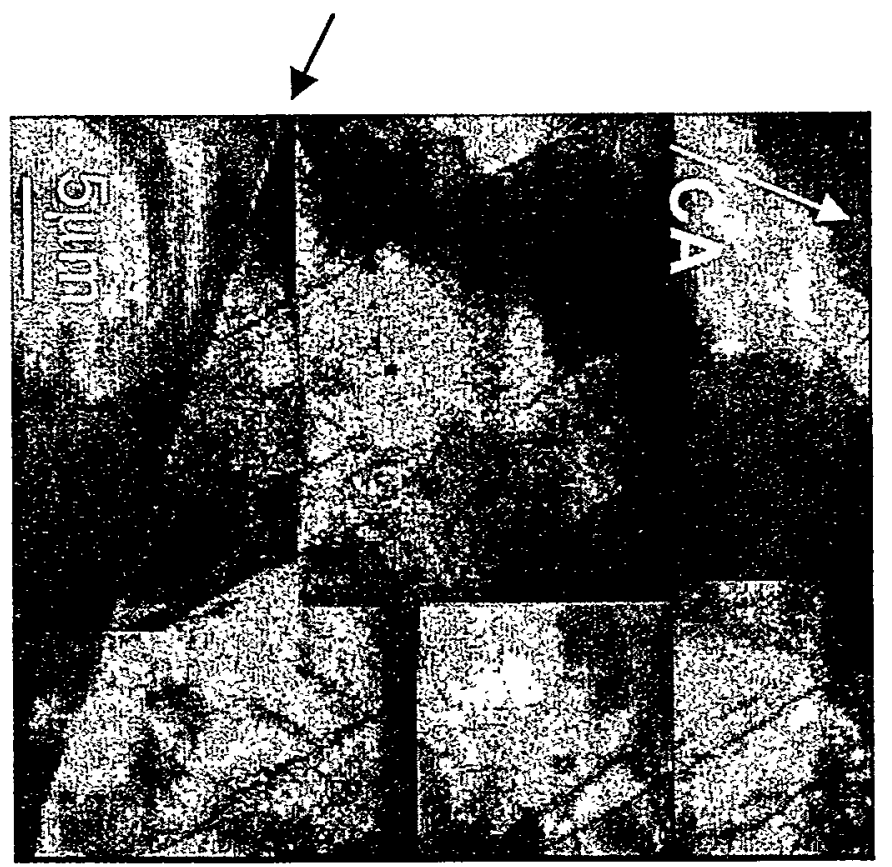




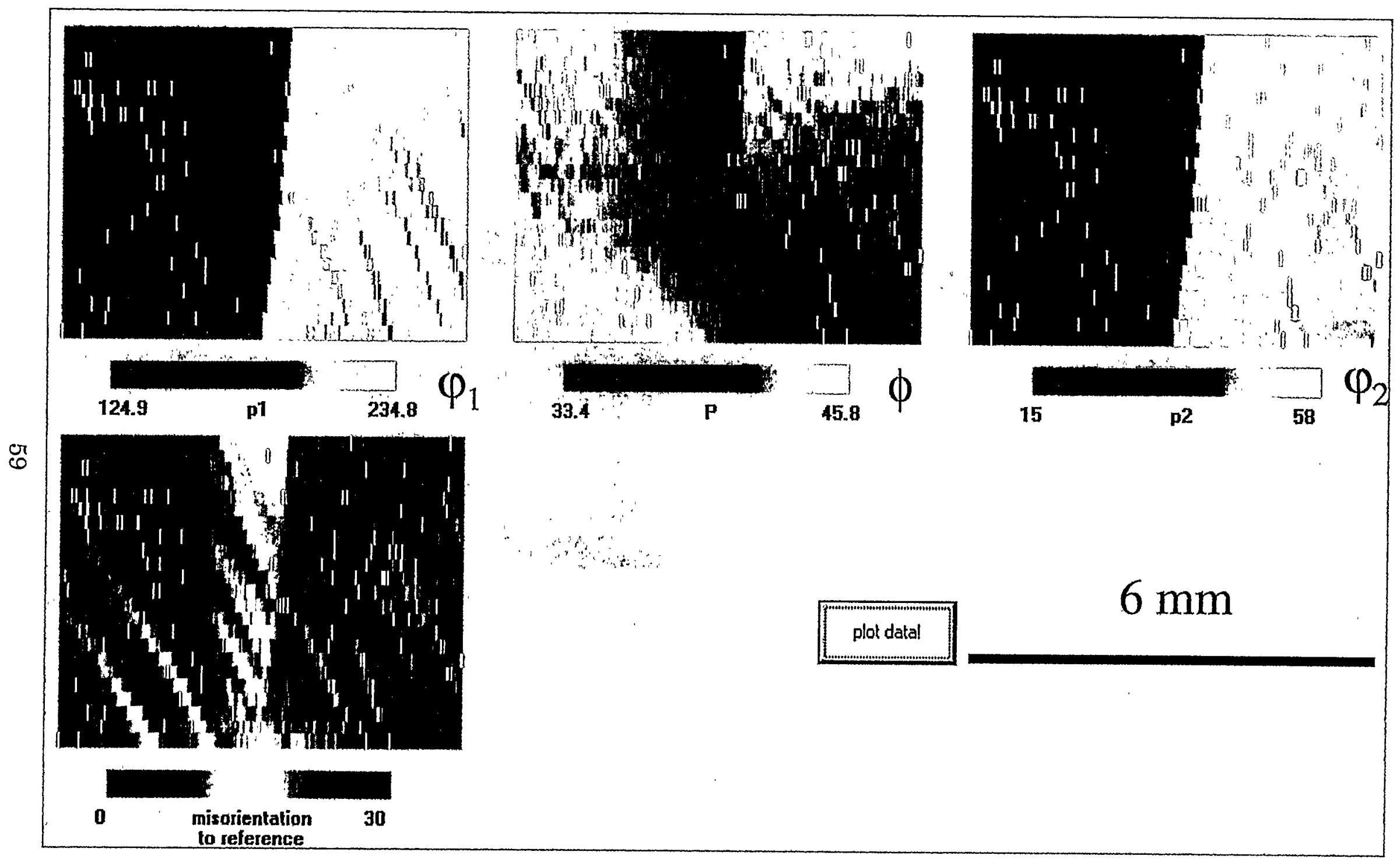

Figure 4-9. a) Local orientations (Euler angles) from EBSP reveal macroscale subdivision of bicrystal following $\varepsilon_{\mathrm{vM}}=0.3$. 







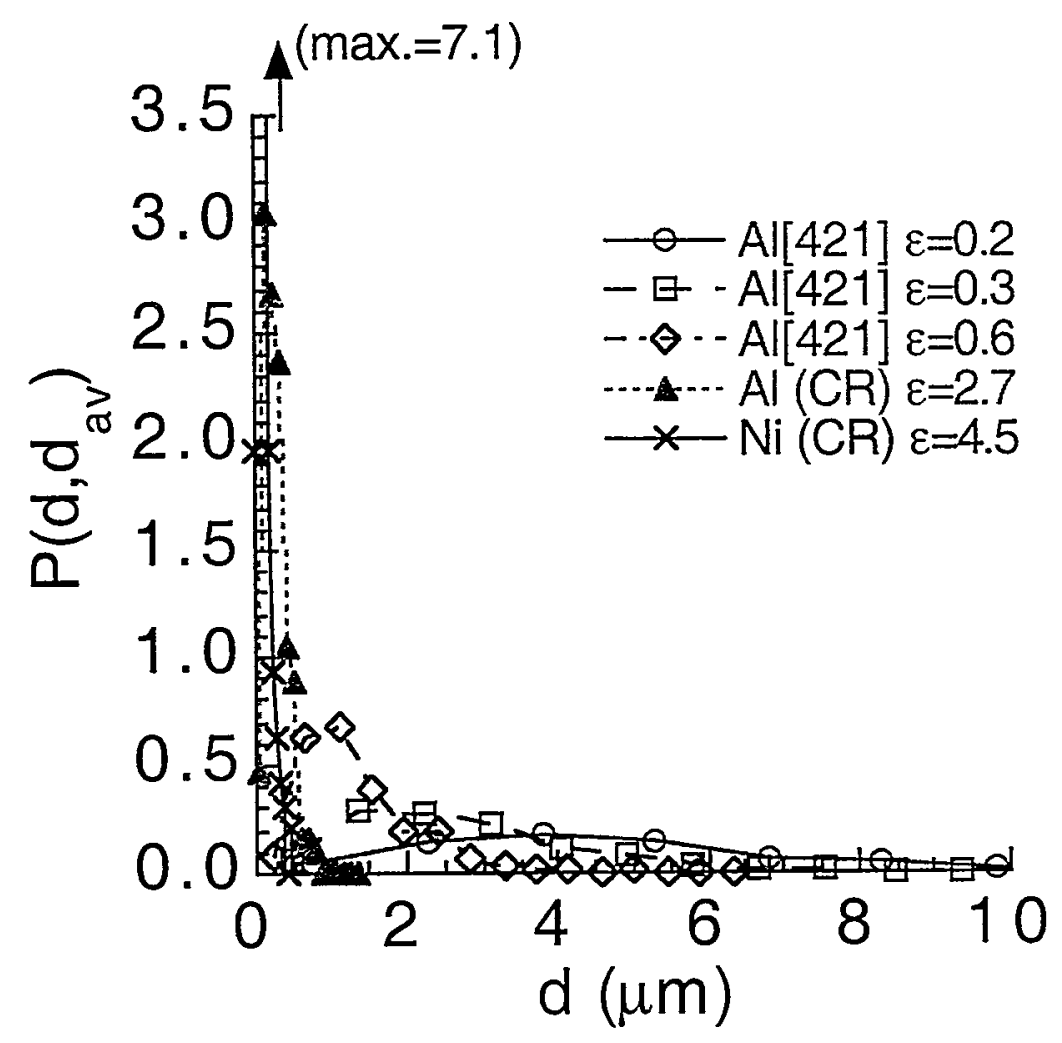

Figure 4-10. Wide range of probability distributions of GNB spacings observed for compressed aluminum single crystals, rolled commercial purity aluminum and rolled high purity nickel (Godfrey and Hughes, 2000). 

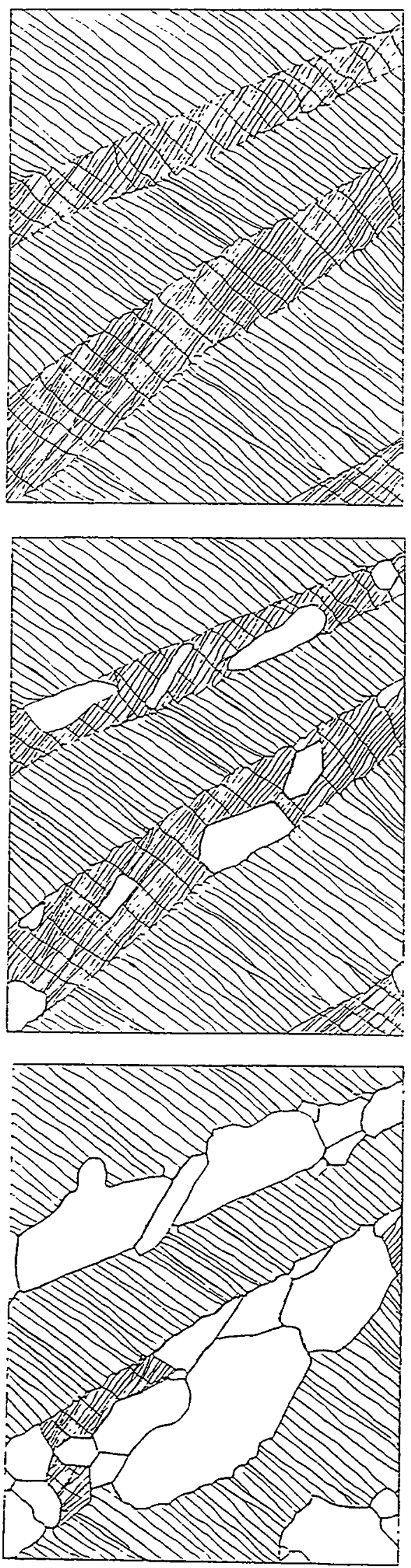

a) Deformed state:

two macroscale bands b) Annealed state first stage: growth of recrystallized grains in macroscale bands c) Annealed state second stage: growth of grains into matrix

Figure 4-11. Schematic drawing showing the two stage recrystallization process in a layered structure. 


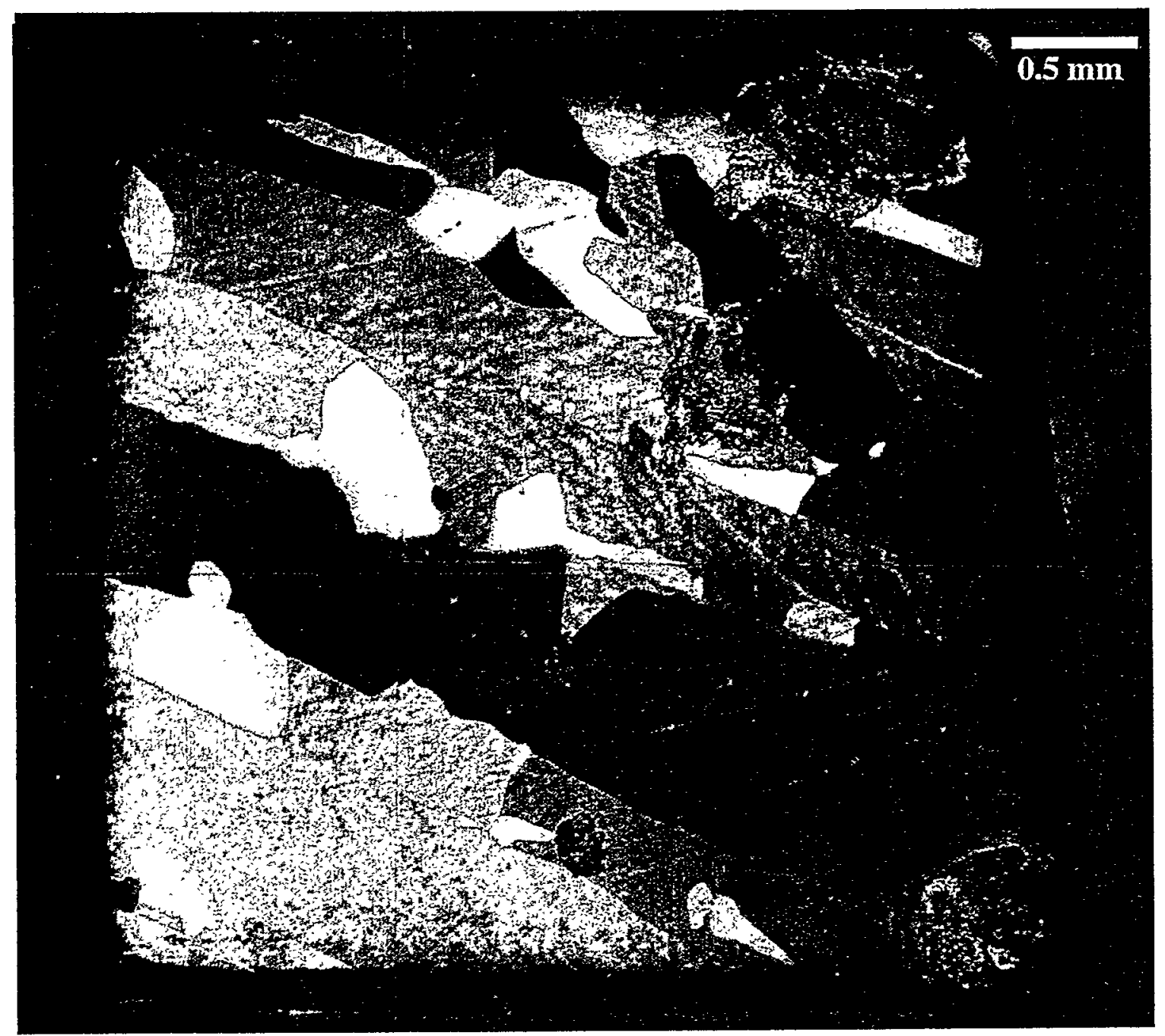

Figure 4-12. Polarized light micrograph showing partial recrystallization along the macroscale bands and SIBM into the matrix regions. Aluminum [421] single crystal compressed to $\varepsilon_{\mathrm{vM}}=0.45$ and subsequently annealed at $150^{\circ} \mathrm{C}$ for ten minutes. 


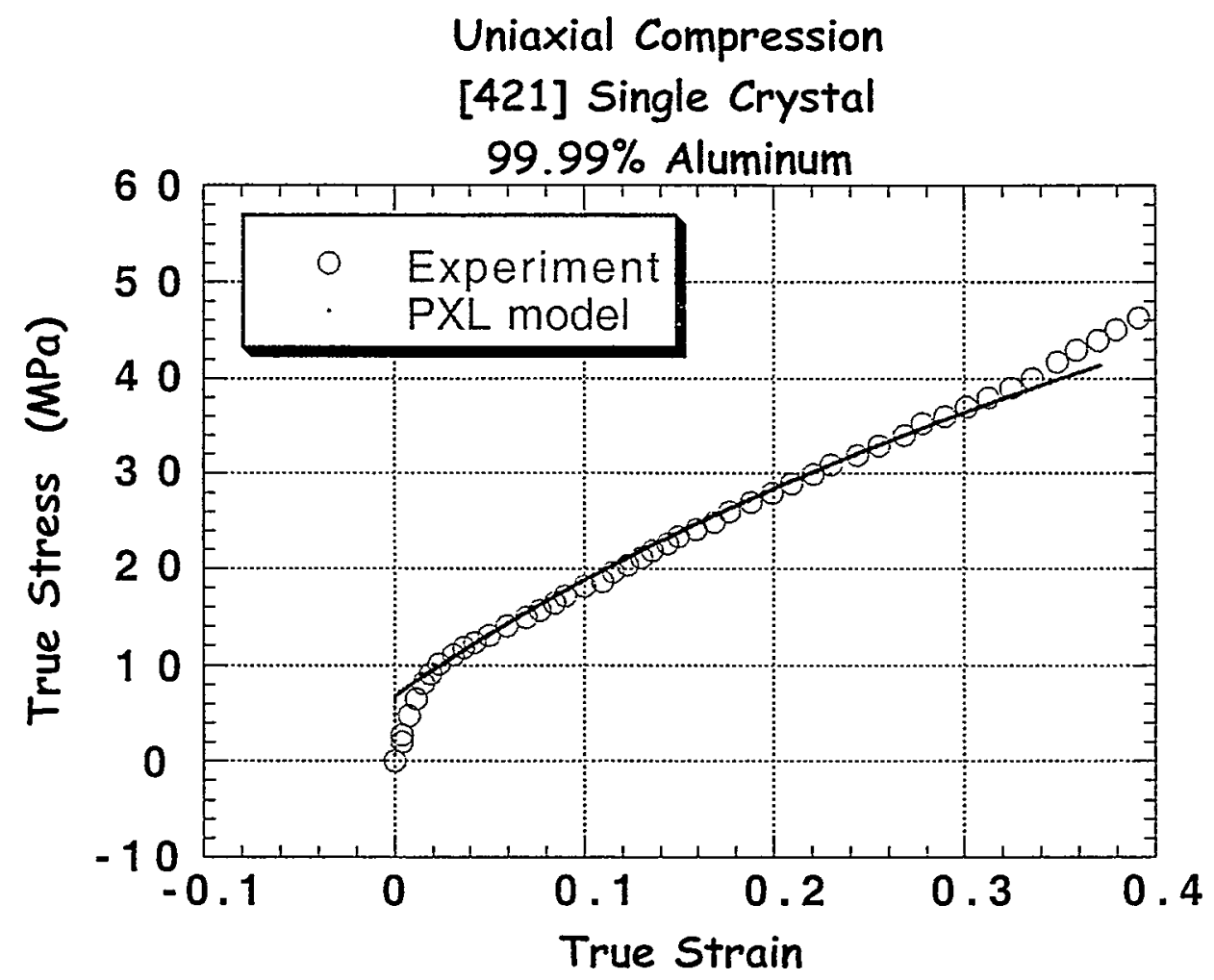

Figure 5-1 Slip system power law constitutive relationship fit to [421] single crystal compression data to $30 \%$ strain. 


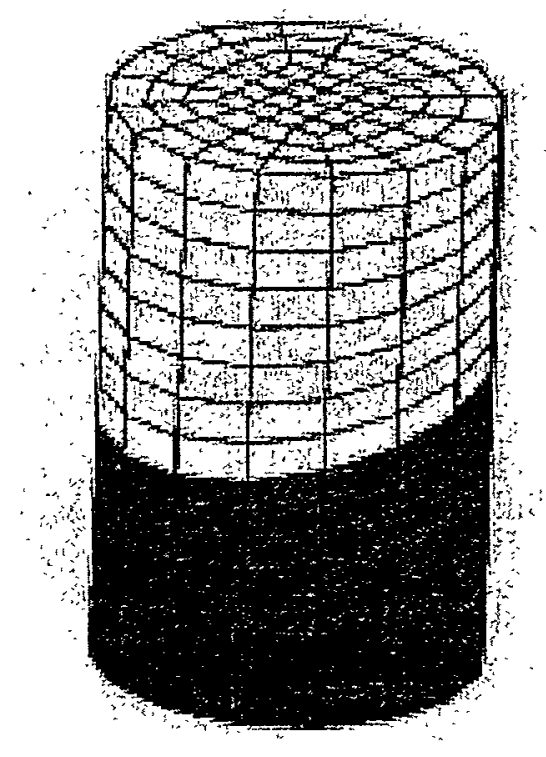

(a)

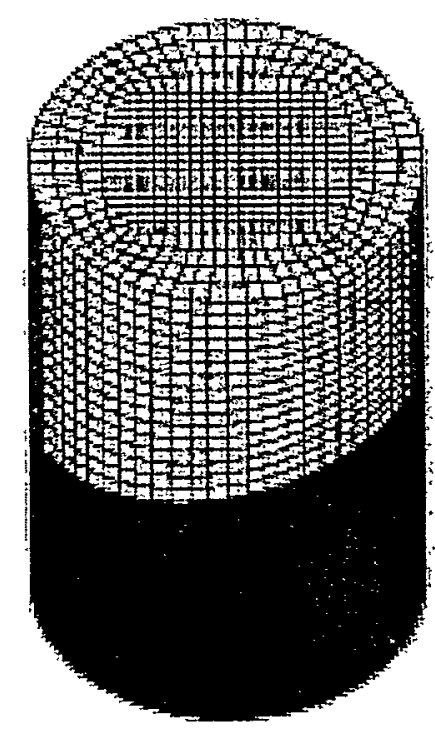

(b)

Figure 5-2. Mesh discretizations for the bicrystal finite element models. The coarse mesh (a) contains 1024 three-dimensional hex elements. The refined mesh (b) contains 16,384 hex elements. Each element is assigned a single crystal orientation initialized to the appropriate individual crystal in which it resides. 

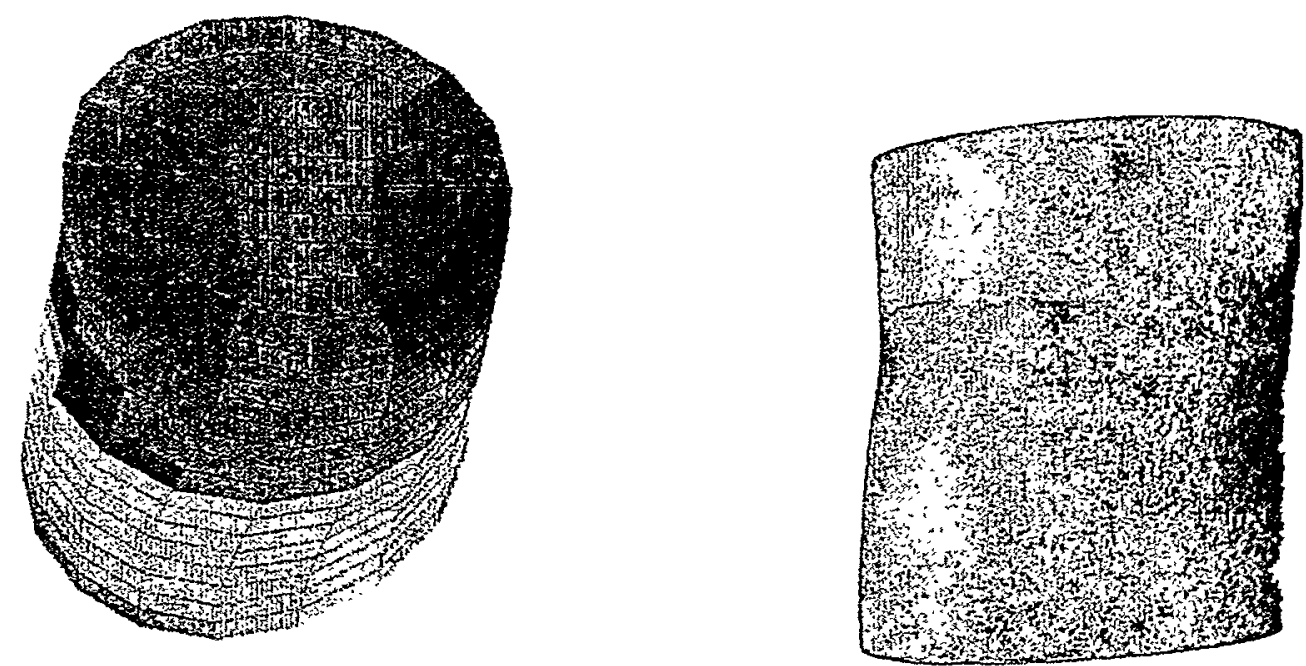

Figure 5-3. The entire bicrystal sample (coarse mesh discretization) captures the overall shape change with no evidence of localized gradients in the deformation field near the bicrystal boundary, $\varepsilon_{\mathrm{vM}}=15 \%$. 

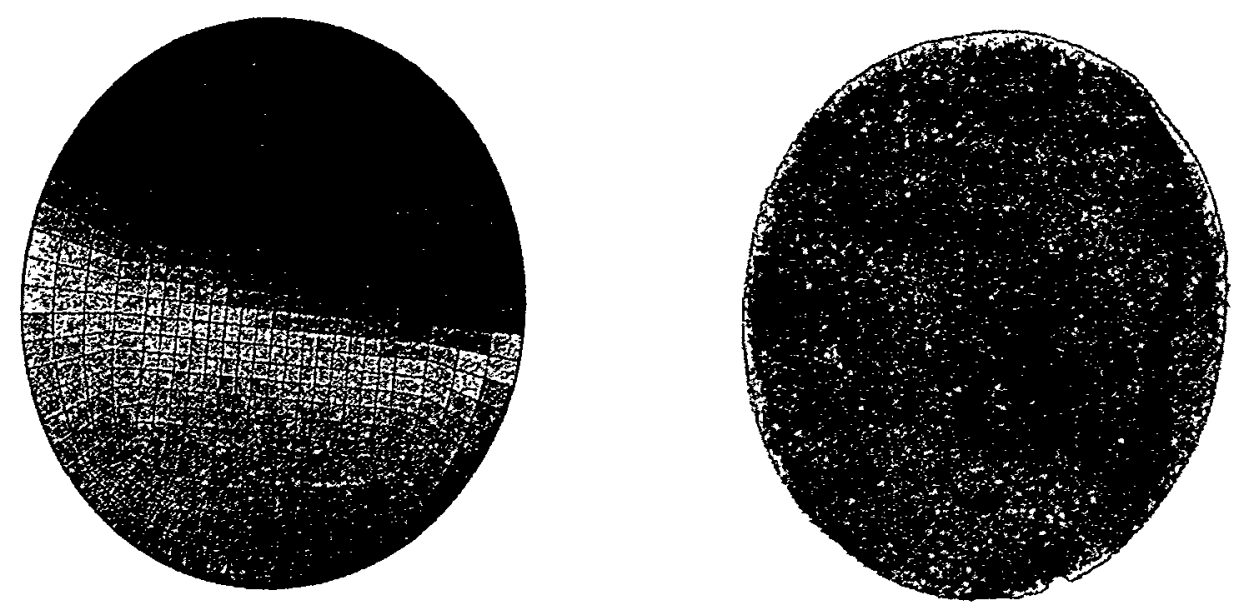

Figure 5-4. Major axis of oval along the projected primary slip direction at the top of an individual crystal for the refined mesh at $15 \%$ strain in compression. 

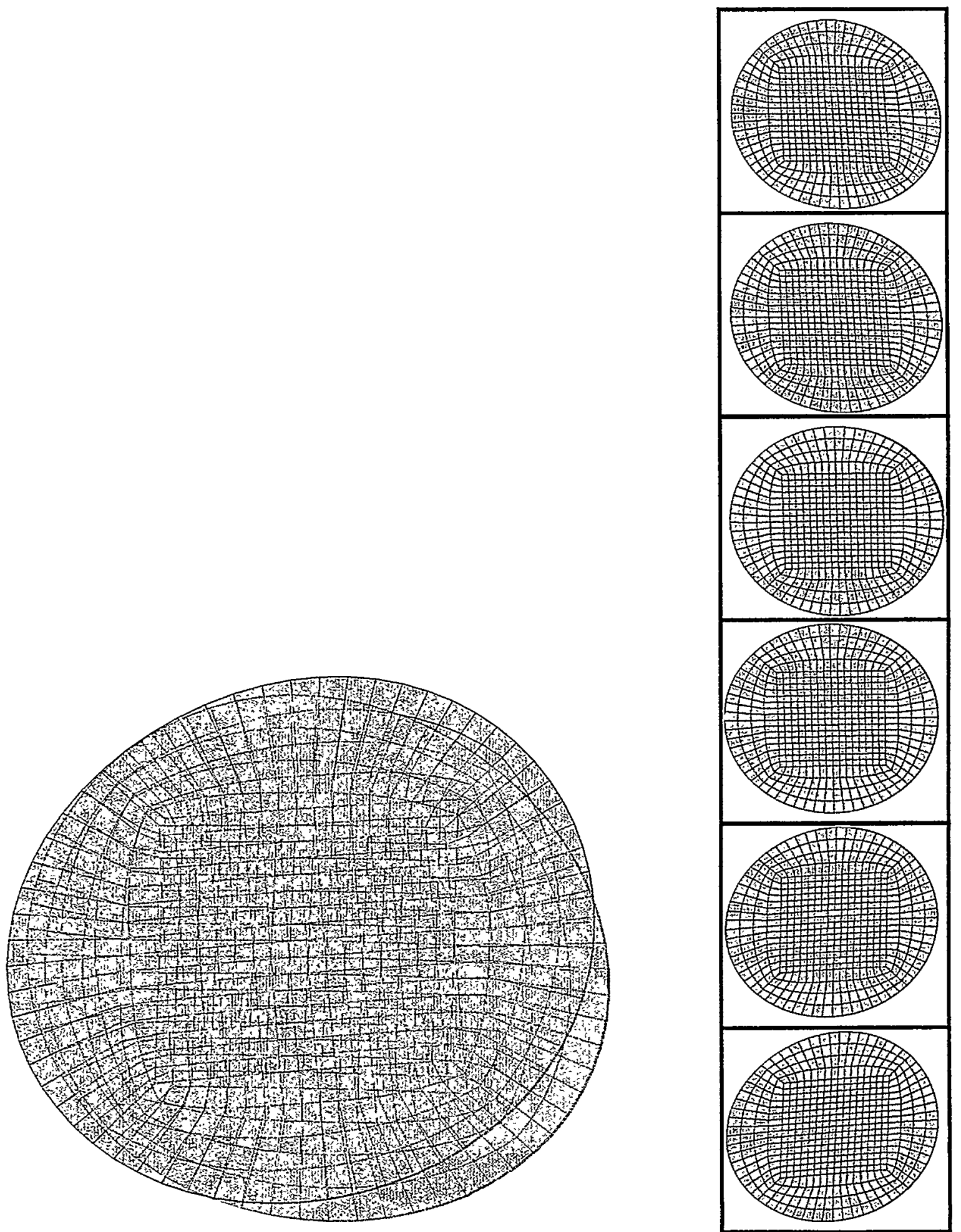

Figure 5-5. Cross sections of the bicrystal FEM mesh following $\varepsilon_{\mathrm{yM}}=0.15$ illustrating that the cross sections with depth develop similarly to the experimental cross-sections shown in Figure 4-3. 


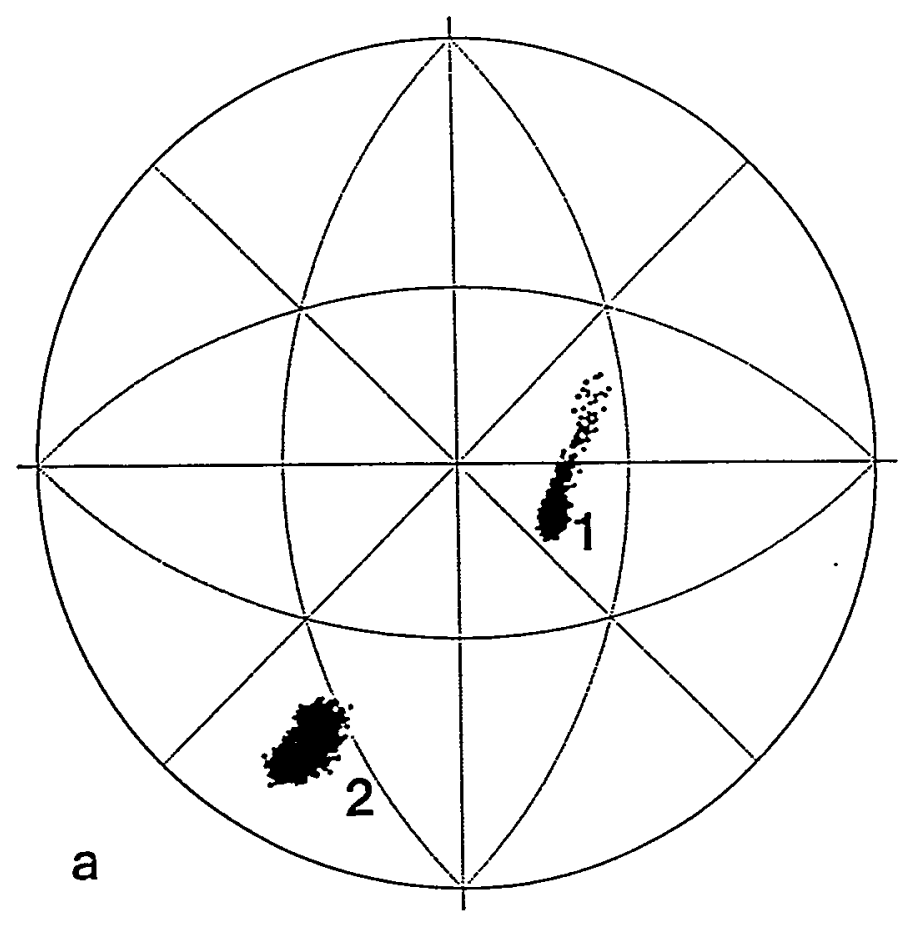

1 , axes for the [421] side of the bicrystal

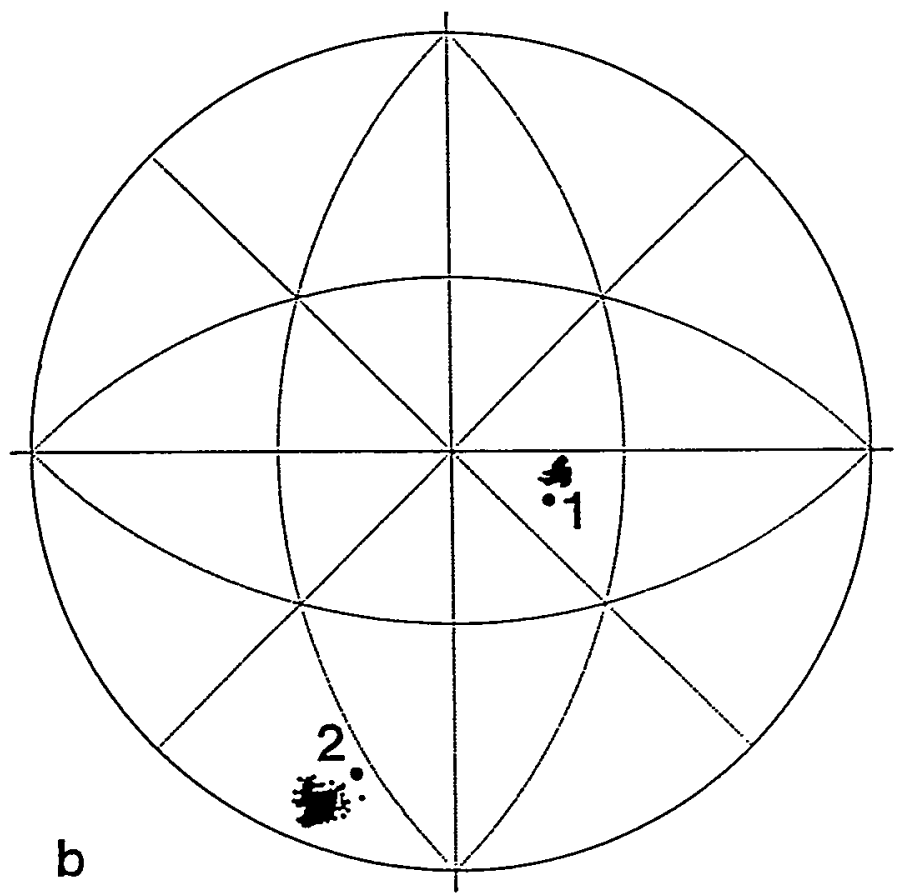

2 , axes for the

[104 $\overline{31}$ 18] side of the bicrystal

Figure 5-6. Inverse pole figures plotted in a (001) stereographic projection a) crystal orientation of the compression axes for the bicrystal experiment, $\varepsilon_{\mathrm{vM}}=0.30 ;$ b) crystal orientation of the compression axes in the crystal plasticity simulation, $\varepsilon_{\mathrm{vM}}=0.15$. 


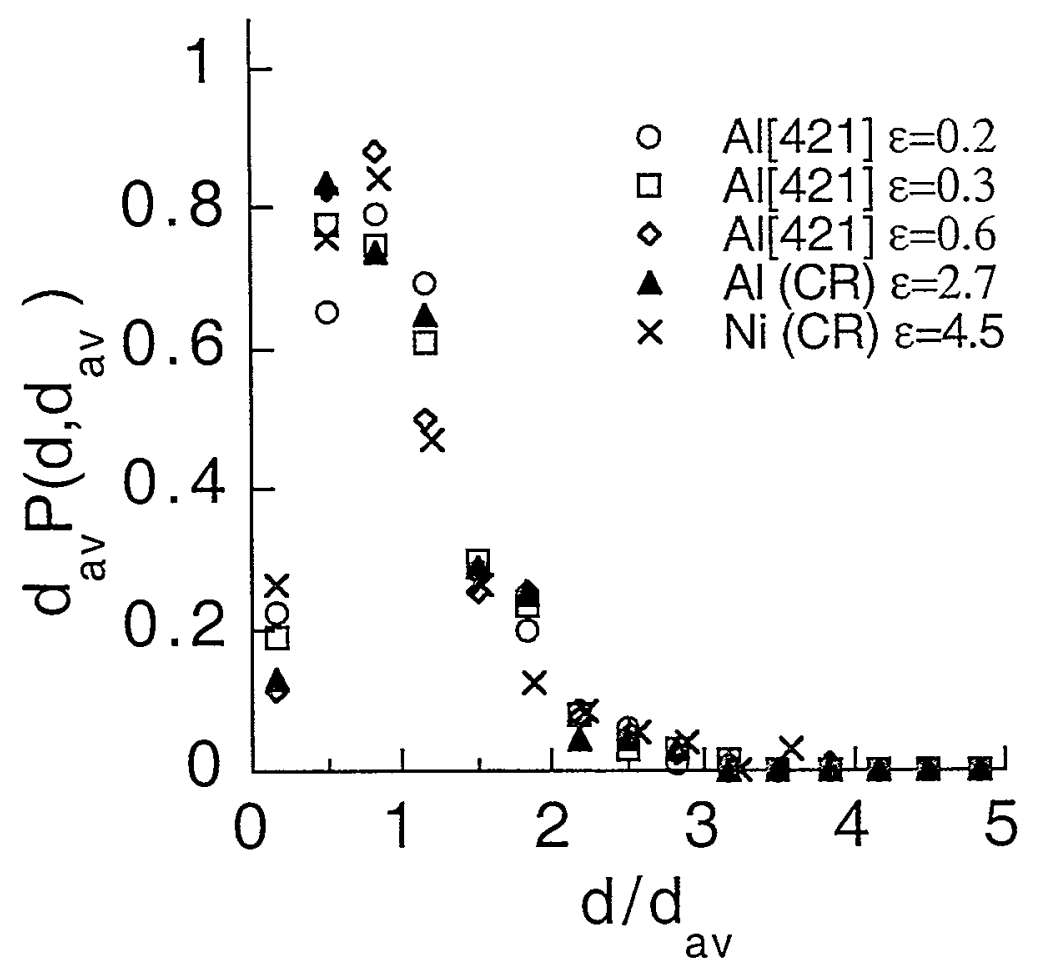

Figure 5-7. Scaled probability distributions for GNB spacing in the [421] single crystal, rolled nickel and aluminum collapse into one function when scaled by the average spacing (Godfrey and Hughes, 2000). 


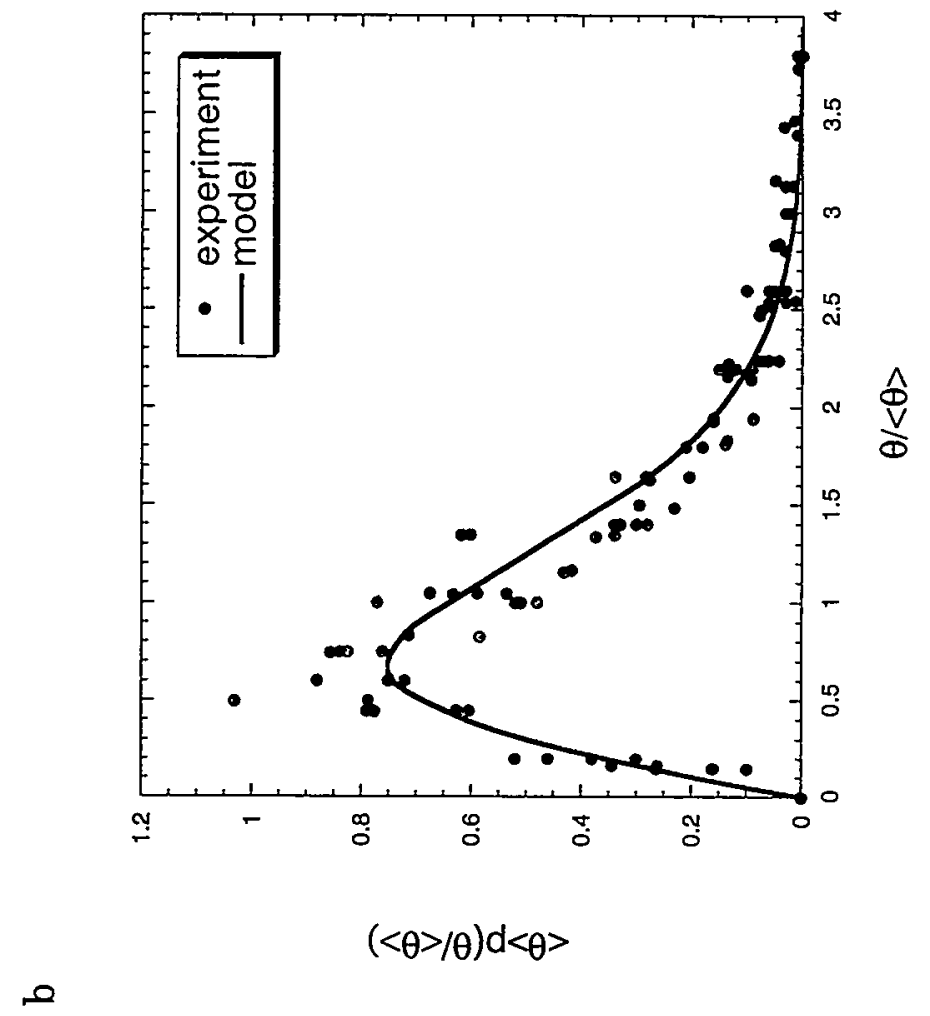

ต

홍

- $\rightarrow$ 过

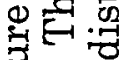

을

ज्ञ

$\oplus$ 영

Z

ర大

얼 오 홀

을

คి

정

형욜

궁

잉

贯 क

동.

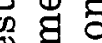

귬류

$0 \dot{1}$

苛贾

엄 곻응

\%

업 유뭉

$\sum$.

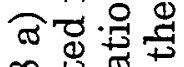

$\infty+\infty$

1

응. 춘

궁 윰

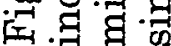

$\varpi$

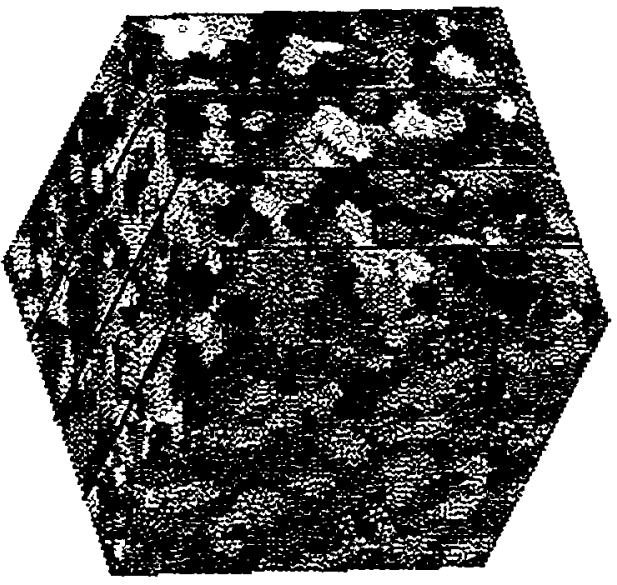




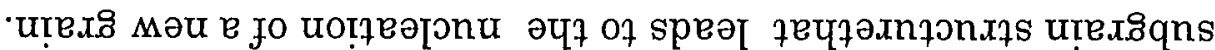

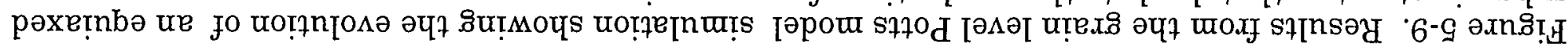
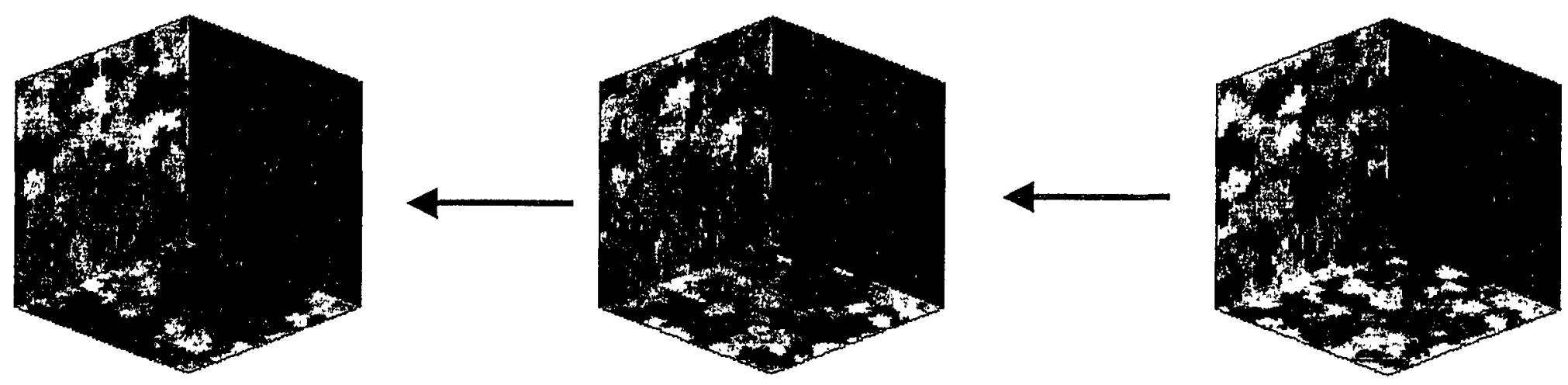


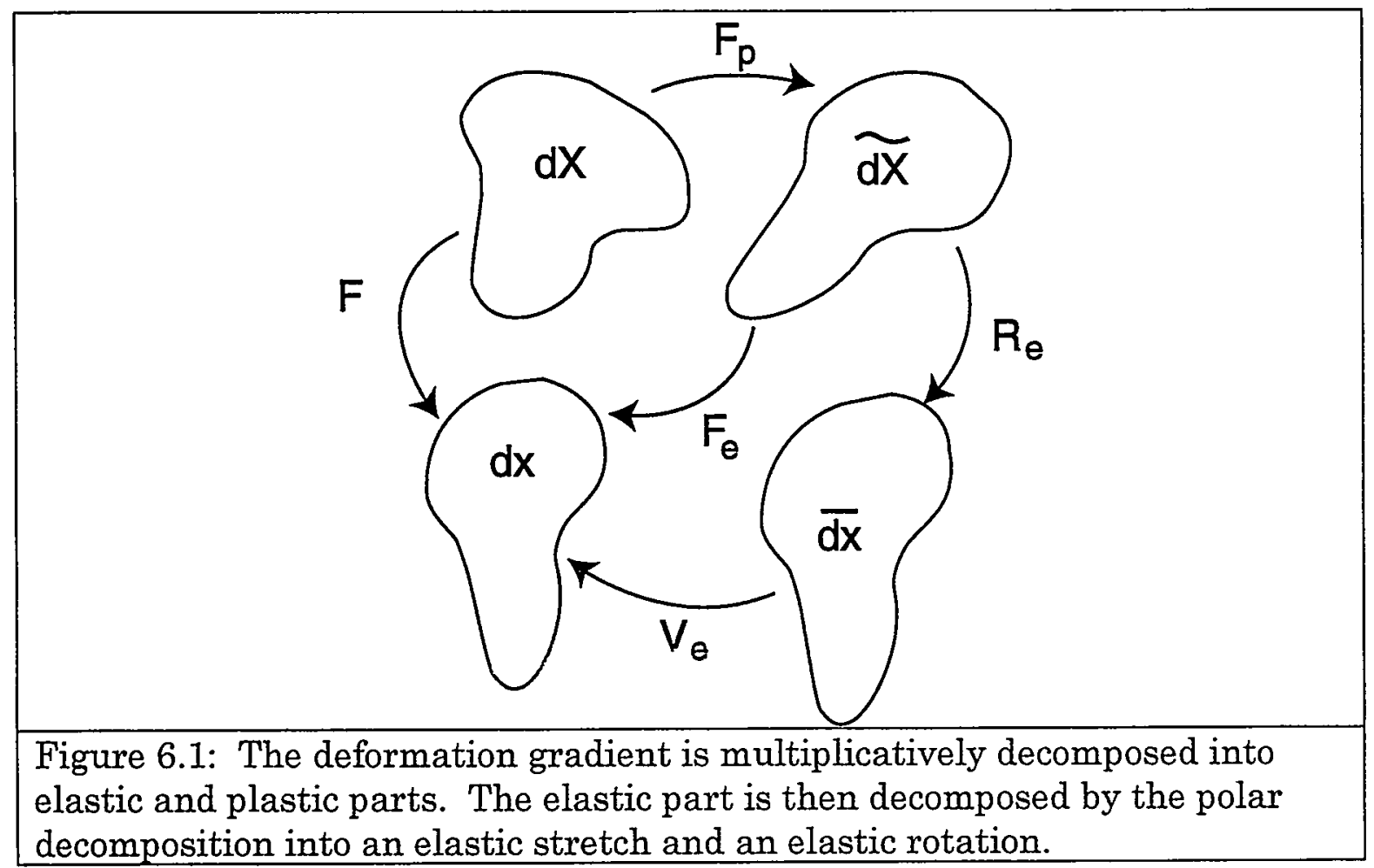



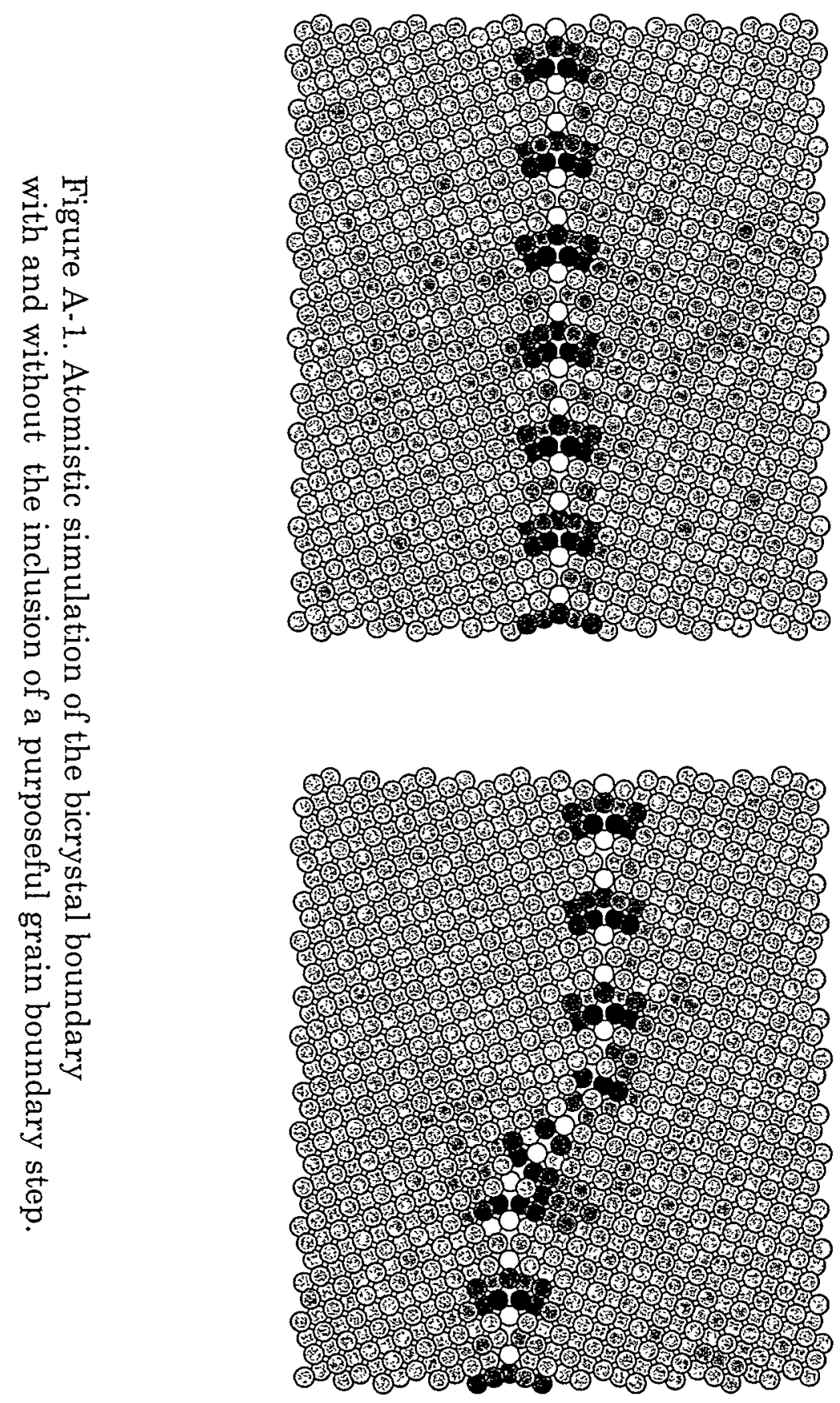
This page intentionally left blank. 


\section{DISTRIBUTION:}

$1 \quad$ Mark. A. Miodownik

Department of Mechanical Engineering

University College Dublin

Dublin 4, IRELAND

1 Daryl C. Chrzan

Materials Ecience and Mineral Engineering

465 Evans Hall \#1760

University of California

Berkeley, California 94720

$1 \quad$ Mark T. Lusk

Division of Engineering

Colorado School of Mines

Golden, Colorado

1 Andrew W. Godfrey,

The Walk

Wootton-by-WOODSTOCK

Oxon,

OX20 1ED

ENGLAND

1 MS 1411 J. M. Philips, 1802

1 MS 1411 E. A. Holm, 1834

1 MS 9001 M. E. John, 8000

Attn: R. C. Wayne, 2200, MS 9005

J. Vitko, 8100, MS 9004

W. J. McLean, 8300, MS 9054

D. R. Henson, 8400, MS 9007

P. N. Smith, 8500, MS 9002

K. E. Washington, 8900, MS 9003

1 MS 9221 C. M. Hartwig, 8510

1 MS 9405 T. M. Dyer, 8700

Attn: R. Q. Hwang, 8721, MS 9161

K. L. Wilson, 8722, MS 9402

J. C. F. Wang, 8723, MS 9403

G. J. Thomas, 8724, MS 9402

W. A. Kawahara, 8725, 9042

J. L. Handrock, 8727, 9042

$1 \quad$ MS $9161 \quad$ D. L. Medlin, 8721 
$10 \quad$ MS 9405

1 MS 9405

$1 \quad$ MS 9161

$1 \quad$ MS 9161

1 MS 9405

$25 \quad$ MS 9405

$1 \quad$ MS 9405

$1 \quad$ MS 9161

$1 \quad$ MS 9405

$5 \quad$ MS 9405

1 MS 9405

$1 \quad$ MS 9042

$1 \quad$ MS 9042

$1 \quad$ MS 9042

1 MS 9012

$1 \quad$ MS 0188

$1 \quad$ MS 0899

1 MS 9021

$1 \quad$ MS 9021

$3 \quad$ MS 9018
D. J. Bammann, 8726

M. C. Bartelt, 8726

E. P. Chen, 8726

S. M. Foiles, 8726

M. F. Horstemeyer, 8726

D. A. Hughes, 8726

R. E. Jones, 8726

P. A. Klein, 8726

E. B. Marin, 8726

V. C. Prantil, 8726

R. A. Regueiro, 8726

M. L. Chiesa, 8727

J. J. Dike, 8727

M. W. Perra, 8728

P. E. Nielan, 8920

D. Chavez, LDRD Office, 4001

Technical Library, 4916

Technical Communications Department, 8528/

Technical Library, MS 0899, 4916

Technical Communications Department, 8528 for DOE/OSTI

Central Technical Files, 8940-2 